\title{
Interactions of renormalized waves in thermalized Fermi-Pasta-Ulam chains
}

\author{
Boris Gershgorin ${ }^{1}$, Yuri V. Lvov ${ }^{1}$ and David $\mathrm{Cai}^{2}$, \\ ${ }^{1}$ Department of Mathematical Sciences, Rensselaer Polytechnic Institute, Troy, NY 12180 \\ ${ }^{2}$ Courant Institute of Mathematical Sciences, New York University, New York, NY 10012
}

\begin{abstract}
The dispersive interacting waves in Fermi-Pasta-Ulam (FPU) chains of particles in thermal equilibrium are studied from both statistical and wave resonance perspectives. It is shown that, even in a strongly nonlinear regime, the chain in thermal equilibrium can be effectively described by a system of weakly interacting renormalized nonlinear waves that possess (i) the Rayleigh-Jeans distribution and (ii) zero correlations between waves, just as noninteracting free waves would. This renormalization is achieved through a set of canonical transformations. The renormalized linear dispersion of these renormalized waves is obtained and shown to be in excellent agreement with numerical experiments. Moreover, a dynamical interpretation of the renormalization of the dispersion relation is provided via a self-consistency, mean-field argument. It turns out that this renormalization arises mainly from the trivial resonant wave interactions, i.e., interactions with no momentum exchange. Furthermore, using a multiple time-scale, statistical averaging method, we show that the interactions of near-resonant waves give rise to the broadening of the resonance peaks in the frequency spectrum of renormalized modes. The theoretical prediction for the resonance width for the thermalized $\beta$-FPU chain is found to be in very good agreement with its numerically measured value.
\end{abstract}

\section{INTRODUCTION}

The study of discrete one-dimensional chains of particles with the nearest-neighbor interactions provides insight to the dynamics of various physical and biological systems, such as crystals, wave systems, and biopolymers $[1-3]$. In the thermal equilibrium state, such nonlinear chains can be described by the canonical Gibbs measure [4] with the Hamiltonian

$$
H=\sum_{j} \frac{p_{j}^{2}}{2}+\frac{\left(q_{j}-q_{j+1}\right)^{2}}{2}+V\left(q_{j}-q_{j+1}\right)
$$

where $p_{j}$ and $q_{j}$ are the momentum and the displacement from the equilibrium position of the $j$-th particle, respectively, $V\left(q_{j}-q_{j+1}\right)$ is the anharmonic part of the potential, and the mass of each particle and the linear spring constant are scaled to unity. In this article, we only consider the potentials of the restoring type, i.e., the potentials for which the Gibbs measure exists. In order to study interactions of waves in such systems, one usually introduces the canonical complex normal variables $a_{k}$ via

$$
a_{k}=\frac{P_{k}-\imath \omega_{k} Q_{k}}{\sqrt{2 \omega_{k}}}
$$

where $P_{k}$ and $Q_{k}$ are the Fourier transforms of $p_{j}$ and $q_{j}$, respectively, and $\omega_{k}=2 \sin (\pi k / N)$ is the linear dispersion relation of the waves represented by $a_{k}$. In terms of the $a_{k}$, the Hamiltonian (1) becomes

$$
H=\sum \omega_{k}\left|a_{k}\right|^{2}+V(a),
$$

where $V(a)$ is the combination of various products of $a_{k}$ and $a_{k}^{*}$ corresponding to various wave-wave interactions.
If the potential in Eq. (1) is harmonic, i.e., $V \equiv 0$, then $a_{k}$ correspond to ideal, free waves, which have no energy exchanges among different $k$ modes. In thermal equilibrium, the Boltzmann distribution $\exp \left(-\theta^{-1} \sum \omega_{k}\left|a_{k}\right|^{2}\right)$ with temperature $\theta$, gives rise to the following properties of free waves

$$
\begin{aligned}
& \left\langle a_{k}^{*} a_{l}\right\rangle=n_{k} \delta_{l}^{k}, \\
& \left\langle a_{k} a_{l}\right\rangle=0,
\end{aligned}
$$

for any $k$ and $l$, where $n_{k} \equiv\left\langle\left|a_{k}\right|^{2}\right\rangle=\theta / \omega_{k}$ is the power spectrum. If the anharmonic part of the potential is sufficiently weak, then corresponding waves $a_{k}$ remain almost free, and Eqs. (4) and (5) would be approximately satisfied in the weakly nonlinear regime. However, when the nonlinearity becomes stronger, waves $a_{k}$ become strongly correlated, and, in general, the correlations between waves [Eq. (5)] no longer vanish. In particular, $\left\langle a_{k} a_{N-k}\right\rangle \neq 0$, as will be shown below. Naturally, the question arises: can the strongly nonlinear system in thermal equilibrium still be viewed as a system of almost free waves in some statistical sense? In this article, we address this question with an affirmative answer: it turns out that the system (1) can be described by a complete set of renormalized canonical variables $\tilde{a}_{k}$, which still possess the wave properties given by Eqs. (4) and (5) with a renormalized linear dispersion. The waves that correspond to these new variables $\tilde{a}_{k}$ will be referred to as renormalized waves. Since these renormalized waves possess the equilibrium Rayleigh-Jeans distribution [5] and vanishing correlations between waves, they resemble free, non-interacting waves, and can be viewed as statistical normal modes. Furthermore, it will be demonstrated that the renormalized linear dispersion for these renormalized waves has the form $\tilde{\omega}_{k}=\eta(k) \omega_{k}$, where $\eta(k)$ is the linear frequency renormalization factor, and is independent of $k$ as a consequence of the Gibbs measure. 
In our method, the construction of the renormalized variables $\tilde{a}_{k}$ does not depend on a particular form or strength of the anharmonic potential, as long as it is of the restoring type with only the nearest neighbor interactions, as in Eq. (1). Therefore, our approach is nonperturbative and can be applied to a large class of systems with strong nonlinearity. However, in this article, we will focus on the $\beta$-FPU chain to illustrate the theoretical framework of the renormalized waves. We will verify that $\tilde{a}_{k}$ effectively constitute normal modes for the $\beta$-FPU chain in thermal equilibrium by showing that (i) the theoretically obtained renormalized linear dispersion relationship is in excellent agreement with its dynamical manifestation in our numerical simulation, and (ii) the equilibrium distribution of $\tilde{a}_{k}$ is still a Rayleigh-Jeans distribution and $\tilde{a}_{k}$ 's are uncorrelated. Note that similar expressions for the renormalization factor $\eta$ have been previously discussed in the framework of an approximate virial theorem [6] or effective long wave dynamics via the Zwanzig-Mori projection [7]. However, in our theory, the exact formula for the renormalization factor is derived from a precise mathematical construction of statistical normal modes, and is valid for all wave modes $k-$ no longer restricted to long waves.

Next, we address how renormalization arises from the dynamical wave interaction in the $\beta$-FPU chain. We will show that the $\beta$-FPU chain can be effectively described as a four-wave interacting Hamiltonian system of the renormalized resonant waves $\tilde{a}_{k}$. We will study the resonance structure of the $\beta$-FPU chain and find that most of the exact resonant interactions are trivial, i.e., the interactions with no momentum exchange among different wave modes. In what follows, the renormalization of the linear dispersion will be explained as a collective effect of these trivial resonant interactions of the renormalized waves $\tilde{a}_{k}$. We will use a self-consistency argument to find an approximation, $\eta_{s c}$, of the renormalization factor $\eta$. As will be seen below, the self-consistency argument essentially is of a mean-field type, i.e., the renormalization arises from the scattering of a wave by a mean-background of waves in thermal equilibrium via trivial resonant interactions. We note that our self-consistency, mean-field argument is not limited to the weak nonlinearity. Very good agreement of the renormalization factor $\eta$ and its dynamical approximation $\eta_{s c}$ - for weakly as well as strongly nonlinear waves - confirms that the renormalization is, indeed, a direct consequence of the trivial resonances.

We will further study the properties of these renormalized waves by investigating how long these waves are coherent, i.e., what their frequency widths are. Therefore, we consider near-resonant interactions of the renormalized waves $\tilde{a}_{k}$, i.e., interactions that occur in the vicinity of the resonance manifold, since most of the exact resonant interactions are trivial, i.e., with no momentum exchanges, and they, cannot effectively redistribute energy among the wave modes.

We will demonstrate that near-resonant interactions of the renormalized waves $\tilde{a}_{k}$ provide a mechanism for effective energy exchanges among different wave modes. Taking into account the near-resonant interactions, we will study analytically the frequency peak broadening of the renormalized waves $\tilde{a}_{k}$ by employing a multiple timescale, statistical averaging method. Here, we will arrive at a theoretical prediction of the spatiotemporal spectrum $\left|\hat{a}_{k}(\omega)\right|^{2}$, where $\hat{a}_{k}(\omega)$ is the Fourier transform of the normal variable $\tilde{a}_{k}(t)$, and $\omega$ is the frequency. The predicted width of frequency peaks is found to be in good agreement with its numerically measured values.

In addition, for a finite $\beta$-FPU chain, we will mention the consequence, to the correlation times of waves, of the momentum exchanges that cross over the first Brillouin zone. This process is known as the umklapp scattering in the setting of phonon scattering [8]. Note that, in the previous studies [9] of the FPU chain from the wave turbulence point of view, the effects arising from the finite nature of the chain were not taken into account, i.e., only the limiting case of $N \rightarrow \infty$, where $N$ is the system size, was considered.

The article is organized as follows. In Section II, we discuss a chain of particles with the nearest-neighbor nonlinear interactions. We demonstrate how to describe a strongly nonlinear system as a system of waves that resemble free waves in terms of the power spectrum and vanishing correlations between waves. We show how to construct the corresponding renormalized variables with the renormalized linear dispersion. In Section III, we rewrite the $\beta$-FPU chain as an interacting four-wave Hamiltonian system. We study the dynamics of the chain numerically and find excellent agreement between the renormalized dispersion, obtained analytically and numerically. In Section IV, we describe the resonance manifold analytically and illustrate its controlling role in long-time averaged dynamics using numerical simulation. In Section V, we derive an approximation for the renormalization factor for the linear dispersion using a self-consistency condition. In Section VI, we study the broadening effect of frequency peaks and predict analytically the form of the spatiotemporal spectrum for the $\beta$-FPU chain. We provide the comparison of our prediction with the numerical experiment. We present the conclusions in Section VII.

\section{RENORMALIZED WAVES}

Consider a chain of particles coupled via nonlinear springs. Suppose the total number of particles is $N$ and the momentum and displacement from the equilibrium position of the $j$-th particle are $p_{j}$ and $q_{j}$, respectively. If only the nearest-neighbor interactions are present, then the chain can be described by the Hamiltonian

$$
H=H_{2}+V,
$$


where the quadratic part of the Hamiltonian takes the form

$$
H_{2}=\frac{1}{2} \sum_{j=1}^{N} p_{j}^{2}+\left(q_{j}-q_{j+1}\right)^{2},
$$

and the anharmonic potential $V$ is the function of the relative displacement $q_{j}-q_{j+1}$. Here periodic boundary conditions $q_{N+1} \equiv q_{1}$ and $p_{N+1} \equiv p_{1}$ are imposed. Since the total momentum of the system is conserved, it can be set to zero.

In order to study the distribution of energy among the wave modes, we transform the Hamiltonian to the Fourier variables $Q_{k}, P_{k}$ via

$$
\left\{\begin{array}{l}
Q_{k}=\frac{1}{\sqrt{N}} \sum_{j=0}^{N-1} q_{j} e^{\frac{2 \pi \imath k j}{N}} \\
P_{k}=\frac{1}{\sqrt{N}} \sum_{j=0}^{N-1} p_{j} e^{\frac{2 \pi \imath k j}{N}}
\end{array}\right.
$$

This transformation is canonical $[10,11]$ and the Hamiltonian (6) becomes

$$
H=\frac{1}{2} \sum_{k=1}^{N-1}\left|P_{k}\right|^{2}+\omega_{k}^{2}\left|Q_{k}\right|^{2}+V(Q),
$$

where $\omega_{k}=2 \sin (\pi k / N)$ is the linear dispersion relation. Note that, throughout the paper, for the simplicity of notation, we denote the periodic wave number space by the set of integers in the range $[0, N-1]$, i.e., we drop the conventional factor, $2 \pi / N$. The zeroth mode vanishes due to the fact that the total momentum is zero.

If the system (9) is in thermal equilibrium, then the canonical Gibbs measure, with the corresponding partition function

$$
Z=\int_{-\infty}^{\infty} e^{-H(p, q) / \theta} d p d q
$$

with the temperature $\theta$, can be used to describe the statistical behavior of the system. We consider the systems with the anharmonic potential of the restoring type, i.e., the potential for which the integral in Eq. (10) converges. It can be easily shown that for system (9) the average kinetic energy $K_{k}$ of each mode is independent of the wave number

$$
\left\langle K_{k}\right\rangle=\left\langle K_{l}\right\rangle,
$$

where $k$ and $l$ are wave numbers, $K_{k} \equiv\left|P_{k}\right|^{2} / 2$, and $\langle\ldots\rangle$ denotes averaging over the Gibbs measure. Similarly, the average quadratic potential $U_{k}$ of each mode is independent of the wave number

$$
\left\langle U_{k}\right\rangle=\left\langle U_{l}\right\rangle,
$$

where $U_{k} \equiv \omega_{k}^{2}\left|Q_{k}\right|^{2} / 2$.
If the nonlinear interactions are weak, then it is convenient to further transform the Hamiltonian (9) to the complex normal variables defined by Eq. (2). This transformation is canonical, i.e., the dynamical equation of motion becomes

$$
\imath \dot{a}_{k}=\frac{\partial H}{\partial a_{k}^{*}} .
$$

In terms of these normal variables, the Hamiltonian (9) takes the form (3). For the system of noninteracting waves, i.e., $H=\sum_{k=1}^{N-1} \omega_{k}\left|a_{k}\right|^{2}$, we obtain a standard virial theorem in the form

$$
\left.\left\langle K_{k}\right\rangle\right|_{V=0}=\left.\left\langle U_{k}\right\rangle\right|_{V=0} .
$$

As a consequence of this virial theorem, we have the properties of free waves, which were already mentioned above [Eqs. (4) and (5)], i.e.,

$$
\begin{aligned}
& \left\langle a_{k}^{*} a_{l}\right\rangle=\frac{1}{2 \omega_{k}}\left(\left\langle\left|P_{k}\right|^{2}\right\rangle+\omega_{k}^{2}\left\langle\left|Q_{k}\right|^{2}\right\rangle\right) \delta_{l}^{k}=\frac{\theta}{\omega_{k}} \delta_{l}^{k}, \\
& \left\langle a_{k} a_{l}\right\rangle=\frac{1}{2 \omega_{k}}\left(\left\langle\left|P_{k}\right|^{2}\right\rangle-\omega_{k}^{2}\left\langle\left|Q_{k}\right|^{2}\right\rangle\right) \delta_{N}^{k+l}=0,
\end{aligned}
$$

for all wave numbers $k$ and $l$. Note that equation (15) gives the classical Rayleigh-Jeans distribution for the power spectrum of free waves [5]

$$
n_{k}=\frac{\theta}{\omega_{k}} .
$$

However, if the nonlinearity is present, the waves $a_{k}$ and $a_{N-k}$ become correlated, i.e.,

$$
\left\langle a_{k} a_{N-k}\right\rangle=\frac{1}{2 \omega_{k}}\left(\left\langle\left|P_{k}\right|^{2}\right\rangle-\omega_{k}^{2}\left\langle\left|Q_{k}\right|^{2}\right\rangle\right) \neq 0,
$$

since the property (14) is no longer valid.

As we mentioned before, a complete set of new renormalized variables $\tilde{a}_{k}$ can be constructed, so that the strongly nonlinear system can be viewed as a system of "free" waves in the sense of vanishing correlations and the power spectrum, i.e., the new variables $\tilde{a}_{k}$ satisfy the properties of free waves given in Eqs. (15) and (16). Next, we show how to construct these renormalized variables $\tilde{a}_{k}$.

Consider the generalization of the transformation (2), namely, the transformation from the Fourier variables $Q_{k}$ and $P_{k}$ to the renormalized variables $\tilde{a}_{k}$ by

$$
\tilde{a}_{k}=\frac{P_{k}-\imath \tilde{\omega}_{k} Q_{k}}{\sqrt{2 \tilde{\omega}_{k}}},
$$

where $\tilde{\omega}_{k}$ is an arbitrary function with the only restrictions

$$
\tilde{\omega}_{k}>0, \quad \tilde{\omega}_{k}=\tilde{\omega}_{N-k} .
$$

One can show that, these restrictions (20) provide a necessary and sufficient condition for the transformation (19) 
to be canonical. For the renormalized waves $\tilde{a}_{k}$, we can compute

$$
\begin{aligned}
& \left\langle\tilde{a}_{k}^{*} \tilde{a}_{l}\right\rangle=\frac{1}{2 \tilde{\omega}_{k}}\left(\left\langle\left|P_{k}\right|^{2}\right\rangle+\tilde{\omega}_{k}^{2}\left\langle\left|Q_{k}\right|^{2}\right\rangle\right) \delta_{l}^{k}, \\
& \left\langle\tilde{a}_{k} \tilde{a}_{l}\right\rangle=\frac{1}{2 \tilde{\omega}_{k}}\left(\left\langle\left|P_{k}\right|^{2}\right\rangle-\tilde{\omega}_{k}^{2}\left\langle\left|Q_{k}\right|^{2}\right\rangle\right) \delta_{N}^{k+l} .
\end{aligned}
$$

Since we have the freedom of choosing any $\tilde{\omega}_{k}$ (with the only restrictions (20)), we can chose $\tilde{\omega}_{k}$ such that $\left\langle\tilde{a}_{k} \tilde{a}_{N-k}\right\rangle$ vanishes. Thus, the renormalized variables $\tilde{a}_{k}$ for a strongly nonlinear system will behave like the bare variables $a_{k}$ for a noninteracting system in terms of vanishing correlations between waves. Therefore, we determine $\tilde{\omega}_{k}$ via

$$
\left\langle\left|P_{k}\right|^{2}\right\rangle-\tilde{\omega}_{k}^{2}\left\langle\left|Q_{k}\right|^{2}\right\rangle=0 .
$$

Note that the requirement (23) has the form of the virial theorem for the free waves but with the renormalized linear dispersion $\tilde{\omega}_{k}$. We rewrite Eq. (23) in terms of the kinetic and quadratic potential parts of the energy of the mode $k$ as

$$
\frac{\tilde{\omega}_{k}}{\omega_{k}}=\sqrt{\frac{\left\langle K_{k}\right\rangle}{\left\langle U_{k}\right\rangle}} .
$$

The in Eqs. (11) and (12) leads to the $k$ independence of the right-hand side of Eq. (24). This allows us to define the renormalization factor $\eta$ for all $k$ 's by

$$
\eta \equiv \frac{\tilde{\omega}_{k}}{\omega_{k}}=\sqrt{\frac{\langle K\rangle}{\langle U\rangle}} .
$$

for dispersion $\omega_{k}$. Here $K=\sum_{k=1}^{N-1} K_{k}$ and $U=$ $\sum_{k=1}^{N-1} U_{k}$ are the kinetic and the quadratic potential parts of the total energy of the system (9), respectively. Note that the way of constructing the renormalized variables $\tilde{a}_{k}$ via the precise requirement of vanishing correlations between waves yields the exact expression for the renormalization factor, which is valid for all wave numbers $k$ and any strength of nonlinearity. The independence of $\eta$ of the wave number $k$ is a consequence of the Gibbs measure. This $k$ independence phenomenon has been observed in previous numerical experiments $[6,12]$. We will elaborate on this point in the results of the numerical experiment presented in Section III.

The immediate consequence of the fact that $\eta$ is independent of $k$ is that the power spectrum of the renormalized waves possesses the precise Rayleigh-Jeans distribution, i.e.,

$$
\tilde{n}_{k}=\frac{\theta}{\tilde{\omega}_{k}}
$$

from Eq. (21), where $\tilde{n}_{k}=\left\langle\left|\tilde{a}_{k}\right|^{2}\right\rangle$. Combining Eqs. (2) and (19), we find the relation between the "bare" waves $a_{k}$ and the renormalized waves $\tilde{a}_{k}$ to be

$$
a_{k}=\frac{1}{2}\left(\sqrt{\eta}+\frac{1}{\sqrt{\eta}}\right) \tilde{a}_{k}+\frac{1}{2}\left(\sqrt{\eta}-\frac{1}{\sqrt{\eta}}\right) \tilde{a}_{N-k} .
$$

Using Eq. (27), we obtain the following form of the power spectrum for the bare waves $a_{k}$

$$
n_{k}=\frac{1}{2}\left(1+\frac{1}{\eta^{2}}\right) \frac{\theta}{\omega_{k}},
$$

which is a modified Rayleigh-Jeans distribution due to the renormalization factor $\left(1+1 / \eta^{2}\right) / 2$. Naturally, if the nonlinearity becomes weak, we have $\eta \rightarrow 1$, and, therefore, all the variables and parameters with tildes reduce to the corresponding "bare" quantities, in particular, $\tilde{\omega}_{k} \rightarrow \omega_{k}, \tilde{a}_{k} \rightarrow a_{k}, \tilde{n}_{k} \rightarrow n_{k}$. It is interesting to point out that, even in a strongly nonlinear regime, the "freewave" form of the Rayleigh-Jeans distribution is satisfied exactly [Eq. (26)] by the renormalized waves. Thus, we have demonstrated that even in the presence of strong nonlinearity, the system in thermal equilibrium can still be viewed statistically as a system of "free" waves in the sense of vanishing correlations between waves and the power spectrum.

Note that, in the derivation of the formula for the renormalization factor [Eq. (25)], we only assumed the nearest-neighbor interactions, i.e., the potential is the function of $q_{j}-q_{j+1}$. One of the well-known examples of such a system is the $\beta$-FPU chain, where only the forth order nonlinear term in $V$ is present. In the remainder of the article, we will focus on the $\beta$-FPU to illustrate the framework of the renormalized waves $\tilde{a}_{k}$.

\section{NUMERICAL STUDY OF THE $\beta$-FPU CHAIN}

Since its introduction in the early 1950s, the study of the FPU lattice [13] has led to many great discoveries in mathematics and physics, such as soliton theory [3]. Being non-integrable, the FPU system also became intertwined with the celebrated Kolmogorov-Arnold-Moser theorem [11]. Here, we extend our results of the thermalized $\beta$-FPU chain, which were briefly reported in [12].

The Hamiltonian of the $\beta$-FPU chain is of the form

$$
H=\sum_{j=1}^{N} \frac{1}{2} p_{j}^{2}+\frac{1}{2}\left(q_{j}-q_{j+1}\right)^{2}+\frac{\beta}{4}\left(q_{j}-q_{j+1}\right)^{4},
$$

where $\beta$ is a parameter that characterizes the strength of nonlinearity.

The canonical equations of motion of the $\beta$-FPU chain are

$$
\begin{aligned}
\dot{q}_{j} & =\frac{\partial H}{\partial p_{j}}=p_{j}, \\
\dot{p}_{j} & =-\frac{\partial H}{\partial q_{j}}=\left(q_{j-1}-2 q_{j}+q_{j+1}\right) \\
& +\beta\left[\left(q_{j+1}-q_{j}\right)^{3}-\left(q_{j}-q_{j-1}\right)^{3}\right] .
\end{aligned}
$$

To investigate the dynamical manifestation of the renormalized dispersion $\tilde{\omega}_{k}$ of $\tilde{a}_{k}$, we numerically integrate 
Eq. (30). Since we study the thermal equilibrium state [14-17] of the $\beta$-FPU chain, we use random initial conditions, i.e., $p_{j}$ and $q_{j}$ are selected at random from the uniform distribution in the intervals $\left(-p_{\max }, p_{\max }\right)$ and $\left(-q_{\max }, q_{\max }\right)$, respectively, with the two constraints that (i) the total momentum of the system is zero and (ii) the total energy of the system $E$ is set to be a specified constant. We have verified that the results discussed in the paper do not depend on details of the initial data. Note that the behavior of $\beta$-FPU for fixed $N$ is fully characterized by only one parameter $\beta E$ [18]. We use the sixth order symplectic Yoshida algorithm [19] with the time step $d t=0.01$, which ensures the conservation of the total system energy up to the ninth significant digit for a runtime $\tau=10^{6}$ time units. In order to confirm that the system has reached the thermal equilibrium state [20], the value of the energy localization [21] was monitored via $L(t) \equiv N \sum_{j=1}^{N} G_{j}^{2} /\left(\sum_{j=1}^{N} G_{j}\right)^{2}$, where $G_{j}$ is the energy of the $j$-th particle defined as

$$
\begin{aligned}
G_{j} & =\frac{1}{2} p_{j}^{2}+\frac{1}{4}\left[\left(q_{j}-q_{j+1}\right)^{2}+\left(q_{j-1}-q_{j}\right)^{2}\right] \\
& +\frac{\beta}{8}\left[\left(q_{j}-q_{j+1}\right)^{4}+\left(q_{j-1}-q_{j}\right)^{4}\right] .
\end{aligned}
$$

If the energy of the system is concentrated around one site, then $L(t)=O(N)$. Whereas, if the energy is uniformly distributed along the chain, then $L(t)=O(1)$. In our simulations, in thermal equilibrium states, $L(t)$ is fluctuating in the range of 1-3. Since our simulation is of microcanonical ensemble, we have monitored various statistics of the system to verify that the thermal equilibrium state that is consistent with the Gibbs distribution (canonical ensemble) has been reached. Moreover, we verified that, for $N$ as small as 32 and up to as large as 1024, the equilibrium distribution in the thermalized state in our microcanonical ensemble simulation is consistent with the Gibbs measure. We compared the renormalization factor (25) by computing the values of $\langle K\rangle$ and $\langle U\rangle$ numerically and theoretically using the Gibbs measure and found the discrepancy of $\eta$ to be within $0.1 \%$ for $\beta=1$ and the energy density $E / N=0.5$ for $N$ from 32 to 1024 .

We now address numerically how the renormalized linear dispersion $\tilde{\omega}_{k}$ manifests itself in the dynamics of the $\beta$-FPU system. We compute the spatiotemporal spectrum $\left|\hat{a}_{k}(\omega)\right|^{2}$, where $\hat{a}_{k}(\omega)$ is the Fourier transform of $\tilde{a}_{k}(t)$. (Note that, for simplicity of notation, we drop a tilde in $\hat{a}_{k}$.) Figure 1 displays the spatiotemporal spectrum of $\tilde{a}_{k}$, obtained from the simulation of the $\beta$-FPU chain for $N=256, \beta=0.5$, and $E=100$. In order to measure the value of $\eta$ from the spatiotemporal spectrum, we use the following procedure. For the fixed wave number $k$, the corresponding renormalization factor $\eta(k)$ is determined by the location of the center of the frequency spectrum $\left|\hat{a}_{k}(\omega)\right|^{2}$, i.e.,

$$
\eta(k)=\frac{\omega_{c}(k)}{\omega_{k}}, \text { with } \omega_{c}(k)=\frac{\int \omega\left|\hat{a}_{k}(\omega)\right|^{2} d \omega}{\int\left|\hat{a}_{k}(\omega)\right|^{2} d \omega} .
$$

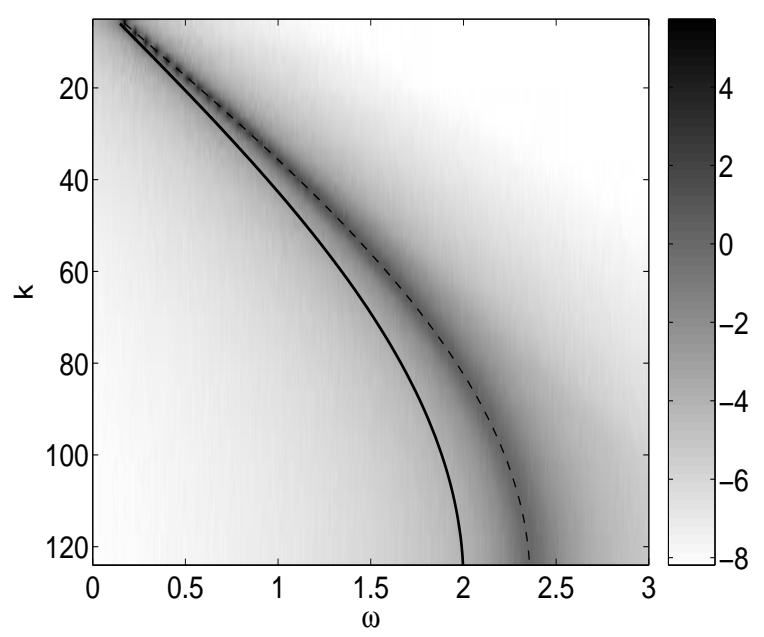

FIG. 1: The spatiotemporal spectrum $\left|\hat{a}_{k}(\omega)\right|^{2}$ in thermal equilibrium. The chain was modeled for $N=256, \beta=0.5$, and $E=100$. $\left[\max \left\{-8, \ln \left|\hat{a}_{k}(\omega)\right|^{2}\right\}\right.$, with corresponding gray scale, is plotted for a clear presentation]. The solid curve corresponds to the usual linear dispersion $\omega_{k}=2 \sin (\pi k / N)$. The dashed curve shows the locations of the actual frequency peaks of $\left|\hat{a}_{k}(\omega)\right|^{2}$.

The renormalization factor $\eta(k)$ of each wave mode $k$ is shown in Fig. 2 (inset). The numerical approximation $\bar{\eta}$ to the value of $\eta$ is obtained by averaging all $\eta(k)$, i.e.,

$$
\bar{\eta}=\frac{1}{N-1} \sum_{k=1}^{N-1} \eta(k) .
$$

The renormalization factor for the case shown in Fig. 1 is measured to be $\bar{\eta} \approx 1.1824$. It can be clearly seen in Fig. 2 (inset) that $\eta(k)$ is nearly independent of $k$ and its variations around $\bar{\eta}$ are less than $0.3 \%$. We also compare the renormalization factor $\eta$ obtained from Eq. (25) (solid line in Fig. 2 (inset)) with its numerically computed approximation $\bar{\eta}$ (dashed line in Fig. 2 (inset)). Equation $(25)$ gives the value $\eta \approx 1.1812$ and the difference between $\eta$ and $\bar{\eta}$ is less then $0.1 \%$, which can be attributed to the statistical errors in the numerical measurement. In Fig. 2, we plot the value of $\eta$ as a function of $\beta$ for the system with $N=256$ particles and the total energy $E=100$. The solid curve was obtained using Eq. (25) while the circles correspond to the value of $\eta$ determined via the numerical spectrum $\left|\hat{a}_{k}(\omega)\right|^{2}$ as discussed above. It can be observed that there is excellent agreement between the theoretic prediction and numerically measured values for a wide range of the nonlinearity strength $\beta$.

In the following Sections, we will discuss how the renormalization of the linear dispersion of the $\beta$-FPU chain in thermal equilibrium can be explained from the wave resonance point of view. In order to give a wave description of the $\beta$-FPU chain, we rewrite the Hamiltonian (29) in terms of the renormalized variables $\tilde{a}_{k}$ [Eq. (19)] with 


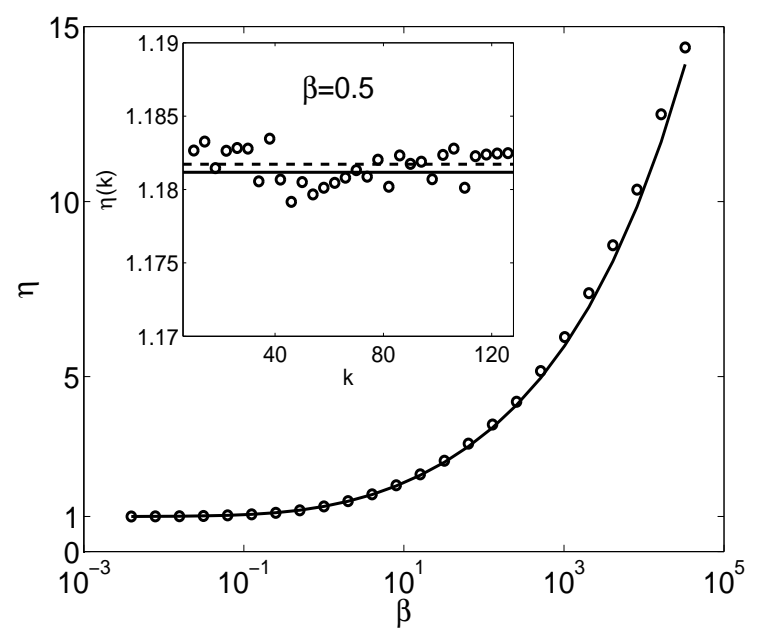

FIG. 2: The renormalization factor as a function of the nonlinearity strength $\beta$. The analytical prediction [Eq. (25)] is depicted with a solid line and the numerical measurement is shown with circles. The chain was modeled for $N=256$, and $E=100$. Inset: Independence of $k$ of the renormalization factor $\eta(k)$. The circles correspond to $\eta(k)$ obtained from the spatiotemporal spectrum shown in Fig. 1 [only even values of $k$ are shown for clarity of presentation]. The dashed line corresponds to the mean value $\bar{\eta}$. For $\beta=0.5$, the mean value of the renormalization factor is found to be $\bar{\eta} \approx 1.1824$. The variations of $\eta_{k}$ around $\bar{\eta}$ are less then $0.3 \%$. [Note the scale of the ordinate.] The solid line corresponds to the renormalization factor $\eta$ obtained from Eq. (25). For the given parameters $\eta \approx 1.1812$.

$\tilde{\omega}_{k}=\eta \omega_{k}$

$$
\begin{aligned}
H & =\sum_{k=1}^{N-1} \frac{\omega_{k}}{2}\left(\eta+\frac{1}{\eta}\right)\left|\tilde{a}_{k}\right|^{2} \\
& +\frac{\omega_{k}}{4}\left(\eta-\frac{1}{\eta}\right)\left(\tilde{a}_{k}^{*} \tilde{a}_{N-k}^{*}+\tilde{a}_{k} \tilde{a}_{N-k}\right) \\
& +\sum_{k, l, m, s=1}^{N-1} T_{m s}^{k l}\left[\Delta_{m s}^{k l} \tilde{a}_{k} \tilde{a}_{l} \tilde{a}_{m}^{*} \tilde{a}_{s}^{*}\right. \\
& +\left(\frac{2}{3} \Delta_{s}^{k l m} \tilde{a}_{k} \tilde{a}_{l} \tilde{a}_{m} \tilde{a}_{s}^{*}+c . c .\right) \\
& \left.+\left(\frac{1}{6} \Delta_{0}^{k l m s} \tilde{a}_{k} \tilde{a}_{l} \tilde{a}_{m} \tilde{a}_{s}+\text { c.c. }\right)\right]
\end{aligned}
$$

where c.c. stands for complex conjugate, and

$$
T_{m s}^{k l}=\frac{3 \beta}{8 N \eta^{2}} \sqrt{\omega_{k} \omega_{l} \omega_{m} \omega_{s}}
$$

is the interaction tensor coefficient. Note that, due to the discrete nature of the system of finite size, the wave space is periodic and, therefore, the "momentum" conservation is guaranteed by the following "periodic" Kronecker delta functions

$$
\begin{aligned}
\Delta_{m s}^{k l} & \equiv \delta_{m s}^{k l}-\delta_{m s}^{k l N}-\delta_{m s N}^{k l} \\
\Delta_{s}^{k l m} & \equiv \delta_{s}^{k l m}-\delta_{s N}^{k l m}+\delta_{s N N}^{k l m} \\
\Delta_{0}^{k l m s} & \equiv \delta_{N N}^{k l m s}-\delta_{N}^{k l m s}-\delta_{N N N}^{k l m s}
\end{aligned}
$$

Here, the Kronecker $\delta$-function is equal to 1 , if the sum of all superscripts is equal to the sum of all subscripts, and 0 , otherwise.

\section{DISPERSION RELATION AND RESONANCES}

In order to address how the renormalized dispersion arises from wave interactions, we study the resonance structure of our nonlinear waves. Since the system (32) is a Hamiltonian system with four-wave interactions, we will discuss the properties of the resonance manifold associated with the $\beta$-FPU system described by Eq. (32) as a first step towards the understanding of its long time statistical behavior. We comment that the resonance structure is one of the main objects of investigation in wave turbulence theory [5, 22-27]. The theory of wave turbulence focuses on the specific type of interactions, namely resonant interactions, which dominate long time statistical properties of the system. On the other hand, the non-resonant interactions are usually shown to have a total vanishing average contribution to a long time dynamics.

In analogy with quantum mechanics, where $a^{+}$and $a$ are creation and annihilation operators, we can view $\tilde{a}_{k}^{*}$ as the outgoing wave with frequency $\tilde{\omega}_{k}$ and $\tilde{a}_{k}$ as the incoming wave with frequency $\tilde{\omega}_{k}$. Then, the nonlinear term $\tilde{a}_{k}^{*} \tilde{a}_{l}^{*} \tilde{a}_{m} \tilde{a}_{s} \Delta_{m s}^{k l}$ in system (32) can be interpreted as the interaction process of the type $(2 \rightarrow 2)$, namely, two outgoing waves with wave numbers $k$ and $l$ are "created" as a result of interaction of the two incoming waves with wave numbers $m$ and $s$. Similarly, $\tilde{a}_{k}^{*} \tilde{a}_{l} \tilde{a}_{m} \tilde{a}_{s} \Delta_{k}^{l m s}$ in system (32) describes the interaction process of the type $(3 \rightarrow 1)$, that is, one outgoing wave with wave number $k$ is "created" as a result of interaction of the three incoming waves with wave numbers $l, m$, and $s$, respectively. Finally, $\tilde{a}_{k} \tilde{a}_{l} \tilde{a}_{m} \tilde{a}_{s} \Delta_{0}^{k l m s}$ describes the interaction process of the type $(4 \rightarrow 0)$, i.e., all four incoming waves interact and annihilate themselves. Furthermore, the complex conjugate terms $\tilde{a}_{k} \tilde{a}_{l}^{*} \tilde{a}_{m}^{*} \tilde{a}_{s}^{*} \Delta_{l m s}^{k}$ and $\tilde{a}_{k}^{*} \tilde{a}_{l}^{*} \tilde{a}_{m}^{*} \tilde{a}_{s}^{*} \Delta_{k l m s}^{0}$ describe the interaction processes of the type $(1 \rightarrow 3)$ and $(0 \rightarrow 4)$, respectively.

Instead of the processes with the "momentum" conservation given via the usual $\delta_{m s}^{k l}, \delta_{s}^{k l m}$, or $\delta_{0}^{k l m s}$ functions for an infinite discrete system, the resonant processes of the $\beta$-FPU chain of a finite size are constrained to the manifold given by $\Delta_{m s}^{k l}, \Delta_{s}^{k l m}$, or $\Delta_{0}^{k l m s}$, respectively. Next, we describe these resonant manifolds in detail. As will be pointed out in Section VI, there is a consequence of this finite size effect to the properties of the renormalized waves. 
The resonance manifold that corresponds to the $(2 \rightarrow$ 2) resonant processes in the discrete periodic system, therefore, is described by

$$
\left\{\begin{array}{l}
k+l \stackrel{N}{=} m+s \\
\tilde{\omega}_{k}+\tilde{\omega}_{l}=\tilde{\omega}_{m}+\tilde{\omega}_{s}
\end{array}\right.
$$

where we have introduced the notation $g \stackrel{N}{=} h$, which means that $g=h, g=h+N$, or $g=h-N$ for any $g$ and $h$. The first equation in system (37) is the "momentum" conservation condition in the periodic wave number space. This "momentum" conservation comes from $\left|\Delta_{m s}^{k l}\right|=1$. (Note that $\left|\Delta_{m s}^{k l}\right|$ can assume only the value of 1 or 0 .) Similarly, from $\left|\Delta_{s}^{k l m}\right|=1$ and $\left|\Delta_{0}^{k l m s}\right|=1$, the resonance manifolds corresponding to the resonant processes of types $(3 \rightarrow 1)$ and $(4 \rightarrow 0)$ are given by

$$
\left\{\begin{array}{l}
k+l+m \stackrel{N}{=} s, \\
\tilde{\omega}_{k}+\tilde{\omega}_{l}+\tilde{\omega}_{m}=\tilde{\omega}_{s},
\end{array}\right.
$$

and

$$
\left\{\begin{array}{l}
k+l+m+s \stackrel{N}{=} 0, \\
\tilde{\omega}_{k}+\tilde{\omega}_{l}+\tilde{\omega}_{m}+\tilde{\omega}_{s}=0,
\end{array}\right.
$$

respectively. For the processes of type $(3 \rightarrow 1)$, the notation $g \stackrel{N}{=} h$ means that $g=h, g=h+N$, or $g=h+2 N$. For the $(4 \rightarrow 0)$ processes, $g \stackrel{N}{=} h$ means that $g=h+N$, $g=h+2 N$, or $g=h+3 N$.

To solve system (37), we rewrite it in a continuous form with $x=k / N, y=l / N, z=m / N, v=s / N$, which are real numbers in the interval $(0,1)$. By recalling that $\tilde{\omega}_{k}=2 \eta \sin (\pi k / N)$, we have

$$
\left\{\begin{array}{l}
x+y \stackrel{1}{=} z+v \\
\sin (\pi x)+\sin (\pi y)=\sin (\pi z)+\sin (\pi v) .
\end{array}\right.
$$

Thus, any rational quartet that satisfies Eq. (40) yields a solution for Eq. (37). There are two distinct types of the solutions of Eq. (40). The first one corresponds to the case

$$
x+y=z+v,
$$

whose only solution is given by

$$
\left\{\begin{array} { l } 
{ x = z , } \\
{ y = v , }
\end{array} \text { or } \quad \left\{\begin{array}{l}
x=v \\
y=z
\end{array}\right.\right.
$$

i.e., these are trivial resonances, as we mentioned above. The second type of the resonance manifold of the $(2 \rightarrow$ 2 )-type interaction processes corresponds to

$$
x+y=z+v \pm 1,
$$

the solution of which can be described by the following two branches

$$
\begin{aligned}
& z_{1}=\frac{x+y}{2}+\frac{1}{\pi} \arcsin (A)+2 j \\
& z_{2}=\frac{x+y}{2}-1-\frac{1}{\pi} \arcsin (A)+2 j
\end{aligned}
$$

where $A \equiv \tan (\pi(x+y) / 2) \cos (\pi(x-y) / 2)$ and $j$ is an integer. The second type of resonances arises from the discreteness of our model of a finite length, leading to non-trivial resonances. For our linear dispersion here, non-trivial resonances are only those resonances that involve wave numbers crossing the first Brillouin zone. As mentioned above, in the setting of the phonon physics, these non-trivial resonant processes are also known as the umklapp scattering processes. In Fig. 3, we plot the solution of Eq. (40) for $x=k / N$ with the wave number $k=90$ for the system with $N=256$ particles (the values of $k$ and $N$ are chosen merely for the purpose of illustration). We stress that all the solutions of the system (40) are given by the Eqs. (41), (42), and (43), and that the non-trivial solutions arise only as a consequence of discreteness of the finite chain. The curves in Fig. 3 represent the loci of $(z, y)$, parametrized by the fourth wave number $v$, i.e., $x, y, z$, and $v$ form a resonant quartet, where $z=m / N$, and $y=l / N$. Note that the fourth wave number $v$ is specified by the "momentum" conservation, i.e., the first equation in Eq. (40). The two straight lines in Fig. 3 correspond to the trivial solutions, as given by Eq. (41). The two curves (dotted and dashed) depict the non-trivial resonances. Note that the dotted part of non-trivial resonance curves corresponds to the branch (42), and the dashed part corresponds to the branch (43), respectively. An immediate question arises: how do these resonant structures manifest themselves in the FPU dynamics in the thermal equilibrium? By examining the Hamiltonian (32), we notice that the resonance will control the contribution of terms like $\tilde{a}_{k}^{*} \tilde{a}_{l}^{*} \tilde{a}_{m} \tilde{a}_{s} \Delta_{m s}^{k l}$ in the long time limit. Therefore, we address the effect of resonance by computing long time average, i.e., $\left\langle\tilde{a}_{k}^{*} \tilde{a}_{l}^{*} \tilde{a}_{m} \tilde{a}_{s}\right\rangle \Delta_{m s}^{k l}$, and comparing this average (Fig. 4) with Fig. 3. To obtain Fig. 4, the $\beta$-FPU system was simulated with the following parameters: $N=256, \beta=0.5, E=\tilde{\tilde{t}}_{1}$, and the averaging time window $\tau=400 \tilde{t}_{1}$, where $\tilde{t}_{1}$ is the longest linear period, i.e., $\tilde{t}_{1}=2 \pi / \tilde{\omega}_{1}$. In Fig. 4 , mode $k$ was fixed with $k=90$ and the mode $s$, a function of $k, l$, and $m$, is obtained from the constraint $k+l \stackrel{N}{=} m+s$, i.e., $\left|\Delta_{m s}^{k l}\right|=1$. Note that we do not impose here the condition $\tilde{\omega}_{k}+\tilde{\omega}_{l}=\tilde{\omega}_{m}+\tilde{\omega}_{s}$, therefore, $\left|\left\langle\tilde{a}_{k}^{*} \tilde{a}_{l}^{*} \tilde{a}_{m} \tilde{a}_{s}\right\rangle \Delta_{m s}^{k l}\right|$ is a function of $l$ and $m$. By comparing Figs. 3 and 4, it can be observed that the locations of the peaks of the long time average $\left|\left\langle\tilde{a}_{k}^{*} \tilde{a}_{l}^{*} \tilde{a}_{m} \tilde{a}_{s}\right\rangle \Delta_{m s}^{k l}\right|$ coincide with the loci of the $(2 \rightarrow 2)$-type resonances. This observation demonstrates that, indeed, there are nontrivial $(2 \rightarrow 2)$-type resonances in the finite $\beta$-FPU chain in thermal equilibrium. Furthermore, it can be observed in Fig. 4 that, in addition to the fact that the resonances manifest themselves as the locations of the peaks of $\left|\left\langle\tilde{a}_{k}^{*} \tilde{a}_{l}^{*} \tilde{a}_{m} \tilde{a}_{s}\right\rangle \Delta_{m s}^{k l}\right|$, the structure of near-resonances is reflected in the finite width of the peaks around the loci of the exact resonances. Note that, due to the discrete nature of the finite $\beta$-FPU system, only those solutions $x, y, z$, and $v$ of Eq. (40), for which $N x, N y, N z$, and $N v$ are integers, yield solutions $k, l$, $m$, and $s$ for Eq. (37). In general, the rigorous treatment 


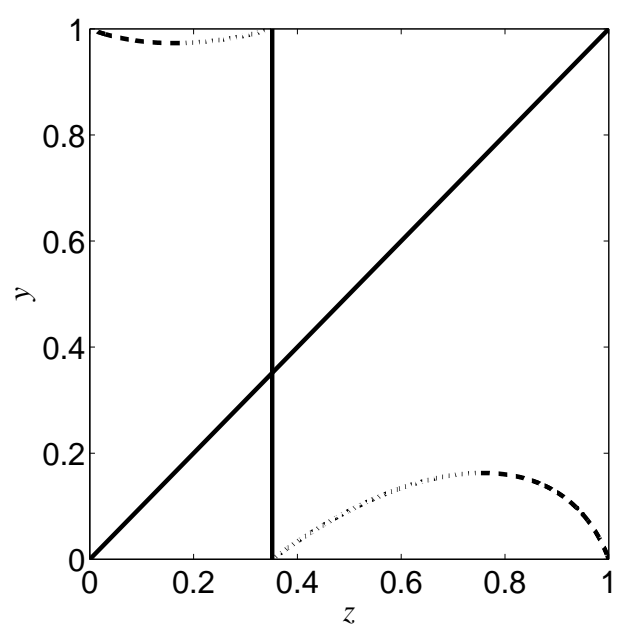

FIG. 3: The solutions of Eq. (40). The solid straight lines correspond to the trivial resonances [solutions of Eq. (41)]. The solutions are shown for fixed $x=k / N, k=90, N=256$ as the fourth wave number $v$ scans from $1 / N$ to $(N-1) / N$ in the resonant quartet Eq. (40). The non-trivial resonances are described by the dotted or dashed curves. The dotted branch of the curves corresponds to the non-trivial resonances described by Eq. (42) and and the dashed branch corresponds to the non-trivial resonances described by Eq. (43).

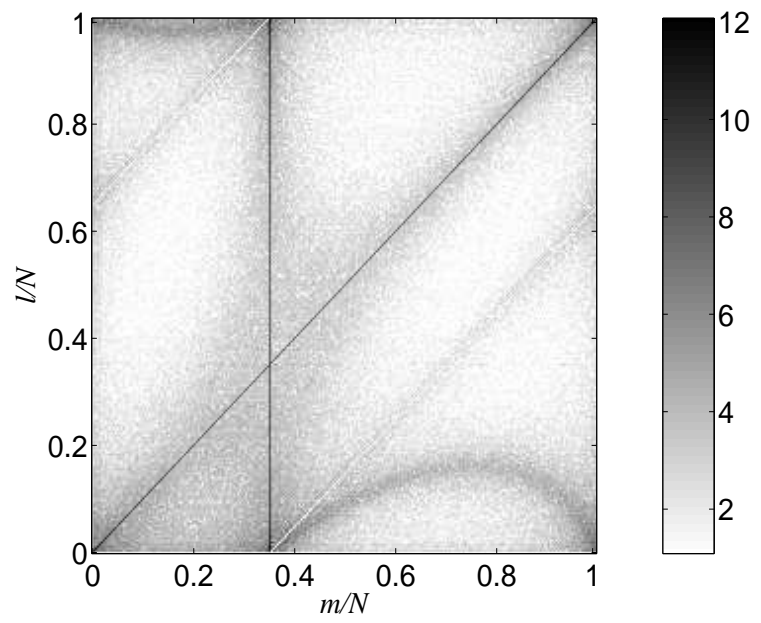

FIG. 4: The long time average $\left|\left\langle\tilde{a}_{k}^{*} \tilde{a}_{l}^{*} \tilde{a}_{m} \tilde{a}_{s}\right\rangle \Delta_{m s}^{k l}\right|$ of the $\beta$-FPU system in thermal equilibrium. The parameters for the FPU chain are $N=256, \beta=0.5$, and $E=100 .\left\langle\tilde{a}_{k}^{*} \tilde{a}_{l}^{*} \tilde{a}_{m} \tilde{a}_{s}\right\rangle \Delta_{m s}^{k l}$ was computed for fixed $k=90$. The darker grayscale corresponds to the larger value of $\left\langle\tilde{a}_{k}^{*} \tilde{a}_{l}^{*} \tilde{a}_{m} \tilde{a}_{s}\right\rangle \Delta_{m s}^{k l}$. The exact solutions of Eq. (40), which are shown in Fig. 3, coincide with the locations of the peaks of $\left|\left\langle\tilde{a}_{k}^{*} \tilde{a}_{l}^{*} \tilde{a}_{m} \tilde{a}_{s}\right\rangle \Delta_{m s}^{k l}\right|$. Therefore, the darker areas represent the near-resonance structure of the finite $\beta$-FPU chain. (The two white lines show the locations, where $s=0$ and, therefore, $\tilde{a}_{k}^{*} \tilde{a}_{l}^{*} \tilde{a}_{m} \tilde{a}_{s} \Delta_{m s}^{k l}=$ 0.) $\left[\max \left\{2, \ln \left(\left|\left\langle\tilde{a}_{k}^{*} \tilde{a}_{l}^{*} \tilde{a}_{m} \tilde{a}_{s}\right\rangle \Delta_{m s}^{k l}\right|\right)\right\}\right.$ with the corresponding grayscale is plotted for a clean presentation]. of the exact integer solutions of Eq. (37) is not straightforward. For example, for $N=256$, we have the following two exact quartets $\vec{k}=\{k, l, m, s\}: \vec{k}=\{k, N / 2-$ $k, N / 2+k, N-k\}, \vec{k}=\{k, N / 2-k, N-k, N / 2+k\}$ for $k<N / 2$, and $\vec{k}=\{k, 3 N / 2-k, k-N / 2, N-k\}$, $\vec{k}=\{k, 3 N / 2-k, N-k, k-N / 2\}$ for $k>N / 2$. We have verified numerically that for $N=256$ there are no other exact integer solutions of Eq. (37). In the analysis of the resonance width in Section VI, we will use the fact that the number of exact non-trivial resonances [Eq. (37)] is significantly smaller than the total number of modes.

The broadening of the resonance peaks in Fig. 4 suggests that, to capture the near-resonances for characterizing long time statistical behavior of the $\beta$-FPU system in thermal equilibrium, instead of Eq. (37), one needs to consider the following effective system

$$
\left\{\begin{array}{l}
k+l \stackrel{N}{=} m+s, \\
\left|\tilde{\omega}_{k}+\tilde{\omega}_{l}-\tilde{\omega}_{m}-\tilde{\omega}_{s}\right|<\Delta \omega,
\end{array}\right.
$$

where $0<\Delta \omega \ll \tilde{\omega}_{k}$ for any $k$, and $\Delta \omega$ characterizes the resonance width, which results from the near-resonace structure. Clearly, $\Delta \omega$ is related to the broadening of the spectral peak of each wave $\tilde{a}_{\alpha}(t)$ with $\alpha=k, l, m$, or $s$ in the quartet, and this broadening effect will be studied in detail in Section VI. Note that the structure of near-resonances is a common characteristic of many periodic discrete nonlinear wave systems [28-30].

Further, it is easy to show that the dispersion relation of the $\beta$-FPU chain does not allow for the occurrence of $(3 \rightarrow 1)$-type resonances, i.e., there are no solutions for Eq. (38), and, therefore, all the nonlinear terms $\tilde{a}_{k}^{*} \tilde{a}_{l} \tilde{a}_{m} \tilde{a}_{s} \Delta_{l m s}^{k}$ are non-resonant and their long time average $\left\langle\tilde{a}_{k}^{*} \tilde{a}_{l} \tilde{a}_{m} \tilde{a}_{s}\right\rangle \Delta_{l m s}^{k}$ vanishes. As for the resonances of type $(4 \rightarrow 0)$, since the dispersion relation is nonnegative, one can immediately conclude that the solution of the system (39) consists only of zero modes. Therefore, the processes of type $(4 \rightarrow 0)$ are also non-resonant, giving rise to $\left\langle\tilde{a}_{k} \tilde{a}_{l} \tilde{a}_{m} \tilde{a}_{s}\right\rangle \Delta_{0}^{k l m s}=0$. In this article, we will neglect the higher order effects of the near-resonances of the types $(3 \rightarrow 1)$ and $(4 \rightarrow 0)$.

In the following sections, we will study the effects of the resonant terms of type $(2 \rightarrow 2)$, namely, the linear dispersion renormalization and the broadening of the frequency peaks of $\tilde{a}_{k}(t)$. It turns out that, the former is related to the trivial resonance of type $(2 \rightarrow 2)$ and the latter is related to the near-resonances, as will be seen below.

\section{SELF-CONSISTENCY APPROACH TO FREQUENCY RENORMALIZATION}

We now turn to the discussion of how the trivial resonances give rise to the dispersion renormalization. This question was examined in [12] before. There, it was shown that the renormalization of the linear dispersion of the $\beta$-FPU chain arises due to the collective effect of 
the nonlinearity. In particular, the trivial resonant interactions of type $(2 \rightarrow 2)$, i.e., the solutions of Eq. (41), enhance the linear dispersion (the renormalized dispersion relation takes the form $\tilde{\omega}_{k}=\eta \omega_{k}$ with $\eta>1$ ), and effectively weaken the nonlinear interactions. Here, we further address this issue and present a self-consistency argument to arrive at an approximation for the renormalization factor $\eta$. As it was mentioned above, the contribution of the non-resonant terms have a vanishing long time effect to the statistical properties of the system, therefore, in our self-consistent approach, we ignore these non-resonant terms. By removing the non-resonant terms and using the canonical transformation

$$
\tilde{a}_{k}=\frac{P_{k}-\imath \eta_{s c} \omega_{k} Q_{k}}{\sqrt{2 \eta_{s c} \omega_{k}}}
$$

where $\eta_{s c}$ is a factor to be determined, we arrive at a simplified effective Hamiltonian from Eq. (32) for the finite $\beta$-FPU system

$$
\begin{aligned}
H_{\mathrm{eff}} & =\sum_{k=1}^{N-1} \frac{\omega_{k}}{2}\left(\eta_{s c}+\frac{1}{\eta_{s c}}\right)\left|\tilde{a}_{k}\right|^{2} \\
& +\sum_{k, l, m, s=1}^{N-1} T_{m s}^{k l} \Delta_{m s}^{k l} \tilde{a}_{k}^{*} \tilde{a}_{l}^{*} \tilde{a}_{m} \tilde{a}_{s} .
\end{aligned}
$$

The "off-diagonal" quadratic terms $\tilde{a}_{k} \tilde{a}_{N-k}$ from Eq. (32) are not present in Eq. (46), since $\tilde{a}_{k}$ are chosen so that $\left\langle\tilde{a}_{k} \tilde{a}_{N-k}\right\rangle=0$ (see Section II). The contribution of the trivial resonances in $H_{\mathrm{eff}}$ is

$$
H_{4}^{\mathrm{tr}}=4 \sum_{k, l=1}^{N-1} T_{k l}^{k l}\left|\tilde{a}_{l}\right|^{2}\left|\tilde{a}_{k}\right|^{2}
$$

which can be "linearized" in the sense that averaging the coefficient in front of $\left|\tilde{a}_{k}\right|^{2}$ in $H_{4}^{\text {tr }}$ gives rise to a quadratic form

$$
H_{2}^{\mathrm{tr}} \equiv \sum_{k=1}^{N-1}\left(4 \sum_{l=1}^{N-1} T_{k l}^{k l}\left\langle\left|\tilde{a}_{l}\right|^{2}\right\rangle\right)\left|\tilde{a}_{k}\right|^{2} .
$$

Note that the subscript 2 in $H_{2}^{\text {tr }}$ emphasizes the fact that $H_{2}^{\text {tr }}$ now can be viewed as a Hamiltonian for the free waves with the familiar effective linear dispersion $\Omega_{k}=$ $4 \sum_{l=1}^{N-1} T_{k l}^{k l}\left\langle\left|\tilde{a}_{l}\right|^{2}\right\rangle[5,12]$. This linearization is essentially a mean-field approximation, since the long-time average of trivial resonances in Eq. (47) is approximated by the interaction of waves $\tilde{a}_{k}$ with background waves $\left\langle\left|\tilde{a}_{l}\right|\right\rangle$. The self consistency condition, which determines $\eta_{s c}$, can be imposed as follows: the quadratic part of the Hamiltonian (46), combined with the "linearized" quadratic part, $H_{2}^{\mathrm{tr}}$, of the quartic $H_{4}^{\mathrm{tr}}$, should be equal to an effective quadratic Hamiltonian $\tilde{H}_{2}=\sum_{k=1}^{N-1} \tilde{\omega}_{k}\left|\tilde{a}_{k}\right|^{2}$ for the renormalized waves, i.e.,

$$
\begin{aligned}
& \sum_{k=1}^{N-1} \frac{\omega_{k}}{2}\left(\eta_{s c}+\frac{1}{\eta_{s c}}\right)\left|\tilde{a}_{k}\right|^{2} \\
& +\sum_{k=1}^{N-1}\left(4 \sum_{l=1}^{N-1} T_{k l}^{k l}\left\langle\left|\tilde{a}_{l}\right|^{2}\right\rangle\right)\left|\tilde{a}_{k}\right|^{2}=\sum_{k=1}^{N-1} \tilde{\omega}_{k}\left|\tilde{a}_{k}\right|^{2},
\end{aligned}
$$

where $\tilde{\omega}_{k}$ is the renormalized linear dispersion, which is used in the definition of our renormalized wave, Eq. (45), and $\tilde{\omega}_{k}=\eta_{s c} \omega_{k}$. Equating the coefficients of $\omega_{k}\left|\tilde{a}_{k}\right|^{2}$ on both sides for every wave number $k$ yields

$$
\frac{1}{2}\left(\eta_{s c}+\frac{1}{\eta_{s c}}\right)+4 \sum_{l=1}^{N-1} \frac{3 \beta}{8 N \eta_{s c}^{2}} \omega_{l}\left\langle\left|\tilde{a}_{l}\right|^{2}\right\rangle=\eta_{s c},
$$

where use is made of Eq. (33). After algebraic simplification, we have the following equation for $\eta_{s c}$

$$
\eta_{s c}^{3}-\eta_{s c}=\frac{3 \beta}{N} \sum_{l=1}^{N-1} \omega_{l}\left\langle\left|\tilde{a}_{l}\right|^{2}\right\rangle .
$$

Using the property (21) of the renormalized normal variables $\tilde{a}_{k}$, we find the following dependence of $\left\langle\left|\tilde{a}_{k}\right|^{2}\right\rangle$ on $\eta_{s c}$

$$
\left\langle\left|\tilde{a}_{l}\right|^{2}\right\rangle=\frac{1}{2 \eta_{s c} \omega_{l}}\left(\left\langle\left|P_{l}\right|^{2}\right\rangle+\eta_{s c}^{2} \omega_{l}^{2}\left\langle\left|Q_{l}\right|^{2}\right\rangle\right)
$$

Combining Eqs. (49) and (50) leads to

$$
\eta_{s c}^{4}-A \eta_{s c}^{2}-B=0
$$

where

$$
\begin{aligned}
& A=1+\frac{3 \beta}{2 N} \sum_{l=1}^{N-1} \omega_{l}^{2}\left\langle\left|Q_{l}\right|^{2}\right\rangle=1+\frac{3 \beta}{N}\langle U\rangle, \\
& B=\frac{3 \beta}{2 N} \sum_{l=1}^{N-1}\left\langle\left|P_{l}\right|^{2}\right\rangle=\frac{3 \beta}{N}\langle K\rangle .
\end{aligned}
$$

The only physically relevant solution of Eq. (51) is

$$
\eta_{s c}=\sqrt{\frac{A+\sqrt{A^{2}+4 B}}{2}} .
$$

The constants $A$ and $B$ can be easily derived using the Gibbs measure.

Next, we compare the renormalization factor $\eta$ [Eq. (25)] with its approximation $\eta_{s c}$ [Eq. (52)] from the self-consistency argument. In Appendix A, we study in detail the behavior of both $\eta$ and $\eta_{s c}$ in the two limiting cases, i.e., when nonlinearity is small $(\beta \rightarrow 0$ with fixed total energy $E)$, and when nonlinearity is large $(\beta \rightarrow \infty$ with fixed total energy $E$ ). As is shown in Appendix A, for the case of small nonlinearity, both $\eta$ and $\eta_{s c}$ have the 


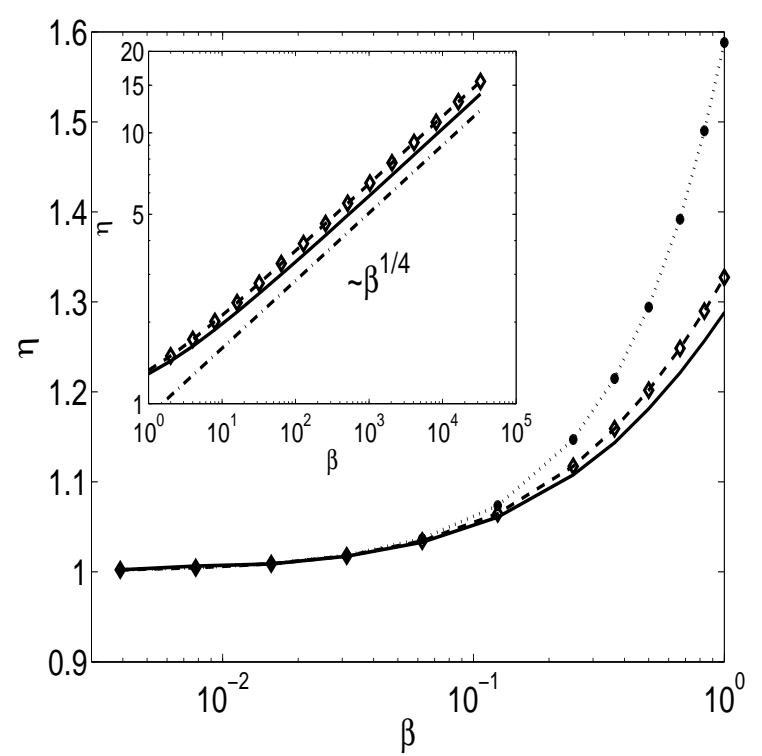

FIG. 5: The renormalization factor as a function of the nonlinearity strength $\beta$ for small values of $\beta$. The renormalization factor $\eta$ [Eq. (25)] is shown with the solid line. The approximation $\eta_{s c}$ [Eq. (52)] (via the self-consistency argument) is depicted with diamonds connected with the dashed line. The small- $\beta$ limit [Eq. (53)] is shown with the solid circles connected with the dotted line. Note that, abscissa is of logarithmic scale. Inset: The renormalization factor as a function of the nonlinearity strength $\beta$ for large values of $\beta$. The renormalization factor $\eta$ [Eq. (25)] is shown with the solid line. $\eta_{s c}$ [Eq.(52)] is depicted with diamonds connected with the dashed line. The large- $\beta$ scaling [Eq. (54)] is shown with the dashed-dotted line. Note that, the plot is of log-log scale with base 10 .

same asymptotic behavior in the first order of the small parameter $\beta$,

$$
\begin{aligned}
\eta & =1+\frac{3 E}{2 N} \beta+O\left(\beta^{2}\right), \\
\eta_{s c} & =1+\frac{3 E}{2 N} \beta+O\left(\beta^{2}\right) .
\end{aligned}
$$

Moreover, in the case of strong nonlinearity $\beta \rightarrow \infty$, both $\eta$ and $\eta_{s c}$ scale as $\beta^{\frac{1}{4}}$, i.e.,

$$
\eta \sim \eta_{s c} \sim \beta^{\frac{1}{4}}
$$

(see Appendix A for details). Note that, in [12], we numerically obtained the scaling $\eta \sim \beta^{0.2}$, which differs from the exact analytical result (54) due to statistical errors in the numerical estimate of the power. In Fig. 5, we plot the renormalization factor $\eta$ and its approximation $\eta_{s c}$ for the case of small nonlinearity $\beta$ for the system with $N=256$ particles and total energy $E=100$. The solid line shows $\eta$ computed via Eq. (25), the diamonds with the dashed line represent the approximation via Eq. (52), and the solid circles with the dotted line correspond to the small- $\beta$ limit (53). In Fig. 5 (inset), we plot the renormalization factor $\eta$ and its approximation $\eta_{s c}$ for the case of large nonlinearity $\beta$ for the system with $N=256$ particles and total energy $E=100$. The solid line shows $\eta$ computed via Eq. (25), the diamonds with the dashed line represent the approximation via Eq. (52), and the dashed-dotted line correspond to the large- $\beta$ scaling (54). Figure 5 shows good agreement between the renormalization factor $\eta$ and its approximation $\eta_{s c}$ from the self consistency argument for a wide range of nonlinearity, from $\beta \sim 10^{-3}$ to $\beta \sim 10^{4}$. This agreement demonstrates, that (i) the effect of the linear dispersion renormalization, indeed, arises mainly from the trivial four-wave resonant interactions, and (ii) our self-consistency, mean-field argument is not restricted to small nonlinearity.

\section{RESONANCE WIDTH}

Finally, we address the question of how coherent these renormalized waves are, i.e., we study how the nonlinear interactions of waves in thermal equilibrium broaden the renormalized dispersion. We will obtain an analytical formula for the spatiotemporal spectrum $\left|\hat{a}_{k}(\omega)\right|^{2}$ for the $\beta$-FPU chain and compare the numerically measured width of the frequency peaks with the predicted width.

In the Hamiltonian (46), the nonlinear terms corresponding to the trivial resonances have been absorbed into the quadratic part via the effective renormalized dispersion $\tilde{\omega}_{k}$. Therefore, the new effective Hamiltonian is

$$
\bar{H}=\sum_{k=1}^{N-1} \tilde{\omega}_{k}\left|\tilde{a}_{k}\right|^{2}+\sum_{k, l, m, s=1}^{N-1} \tilde{T}_{m s}^{k l} \Delta_{m s}^{k l} \tilde{a}_{k}^{*} \tilde{a}_{l}^{*} \tilde{a}_{m} \tilde{a}_{s}
$$

where

$$
\left\{\begin{array}{l}
\tilde{T}_{m s}^{k l}=T_{m s}^{k l}=\frac{3 \beta}{8 N \eta^{2}} \sqrt{\omega_{k} \omega_{l} \omega_{m} \omega_{s}}, k \neq m, \text { and } k \neq s \\
\tilde{T}_{m s}^{k l}=0, \text { otherwise. }
\end{array}\right.
$$

The new interaction coefficient $\tilde{T}_{m s}^{k l}$ ensures that the terms that correspond to the interactions with trivial resonances are not doubly counted in the Hamiltonian (55) and are removed from the quartic interaction. This new interactions in the quartic terms include the exact nontrivial resonant and non-trivial near-resonant as well as non-resonant interactions of the $(2 \rightarrow 2)$-type.

We change the variables to the interaction picture by defining the corresponding variables $b_{k}$ via

$$
b_{k}=\tilde{a}_{k} e^{\imath \tilde{\omega}_{k} t},
$$

so that, the dynamics governed by the Hamiltonian (55) takes the familiar form

$$
i \dot{b}_{k}=2 \sum_{l, m, s=1}^{N-1} \tilde{T}_{m s}^{k l} \Delta_{m s}^{k l} b_{l}^{*} b_{m} b_{s} e^{\tilde{\omega}_{m s}^{k l} t},
$$

where $\tilde{\omega}_{m s}^{k l}=\tilde{\omega}_{k}+\tilde{\omega}_{l}-\tilde{\omega}_{m}-\tilde{\omega}_{s}$ [23]. Without loss of generality, we consider only the case of $k<N / 2$. As we have 
noted before, only for a very small number of quartets does $\tilde{\omega}_{m s}^{k l}$ vanish exactly, i.e., $\tilde{\omega}_{m s}^{k l}=0$. We separate the terms on the RHS of Eq. (57) into two kinds - the first kind with $\tilde{\omega}_{m s}^{k l}=0$ that corresponds to exact non-trivial resonances, and the second kind that corresponds to nontrivial near-resonances and non-resonances. Since, in the summation, the first kind contains far fewer terms than the second kind, and all the terms are of the same order of magnitude, we will neglect the first kind in our analysis. Therefore, Eq. (57) becomes

$$
\imath \dot{b}_{k}=2 \sum_{l, m, s=1}^{N-1} \tilde{T}_{m s}^{k l} \Delta_{m s}^{k l} b_{l}^{*} b_{m} b_{s} e^{\imath \tilde{\omega}_{m s}^{k l} t},
$$

where the prime denotes the summation that neglects the exact non-trivial resonances.

The problem of broadening of spectral peaks now becomes the study of the frequency spectrum of the dynamical variables $b_{k}(t)$ in thermal equilibrium. This is equivalent to study the two-point correlation in time of $b_{k}(t)$

$$
C_{k}(t)=\left\langle b_{k}(t) b_{k}^{*}(0)\right\rangle
$$

where the angular brackets denote the thermal average, since, by Wiener-Khinchin theorem, the frequency spectrum

$$
|b(\omega)|^{2}=\mathfrak{F}^{-1}[C(t)](\omega),
$$

where $\mathfrak{F}^{-1}$ is the inverse Fourier transform in time. Under the dynamics (58), time derivative of the two-point correlation becomes

$$
\begin{aligned}
\dot{C}_{k}(t) & =\left\langle\dot{b}_{k}(t) b_{k}^{*}(0)\right\rangle \\
& =\left\langle-2 \imath \sum_{l, m, s}^{\prime} \tilde{T}_{m s}^{k l} b_{l}^{*}(t) b_{m}(t) b_{s}(t) e^{\imath \tilde{\omega}_{m s}^{k l} t} \Delta_{m s}^{k l} b_{k}^{*}(0)\right\rangle \\
& =-2 \imath \sum_{l, m, s}^{\prime} \tilde{T}_{m s}^{k l} e^{\imath \tilde{\omega}_{m s}^{k l} t} J_{m s}^{k l}(t) \Delta_{m s}^{k l}
\end{aligned}
$$

where

$$
J_{m s}^{k l}(t) \equiv\left\langle b_{l}^{*}(t) b_{k}^{*}(0) b_{m}(t) b_{s}(t)\right\rangle .
$$

In order to obtain a closed equation for $C_{k}(t)$, we need to study the evolution of the fourth order correlator $J_{m s}^{k l}(t)$. We utilize the weak effective nonlinearity in Eq. (55) [12] as the small parameter in the following perturbation analysis and obtain a closure for $C_{k}(t)$, similar to the traditional way of deriving kinetic equation, as in $[5,31]$. We note that the effective interactions of renormalized waves can be weak, as we have shown in [12], even if the $\beta$-FPU chain is in a strongly nonlinear regime. Our perturbation analysis is a multiple time-scale, statistical averaging method. Under the near-Gaussian assumption, which is applicable for the weakly nonlinear wave fields in thermal equilibrium, for the four-point correlator, we obtain

$$
J_{m s}^{k l}(t) \Delta_{m s}^{k l}=C_{k}(t) C_{l}(0)\left(\delta_{m}^{k} \delta_{s}^{l}+\delta_{s}^{k} \delta_{m}^{l}\right) .
$$

Combining Eqs. (56) and (62), we find that the righthand side of Eq. (61) vanishes because

$$
\tilde{T}_{m s}^{k l} J_{m s}^{k l}(t) \Delta_{m s}^{k l}=0 .
$$

Therefore, we need to proceed to the higher order contribution of $J_{m s}^{k l}(t)$. Taking its time derivative yields

$$
\begin{aligned}
\dot{J}_{m s}^{k l}(t) \Delta_{m s}^{k l} & =\left\langle\left[\dot{b}_{l}^{*}(t) b_{m}(t) b_{s}(t)+b_{l}^{*}(t) \dot{b}_{m}(t) b_{s}(t)\right.\right. \\
& \left.\left.+b_{l}^{*}(t) b_{m}(t) \dot{b}_{s}(t)\right] b_{k}^{*}(0)\right\rangle \Delta_{m s}^{k l} .
\end{aligned}
$$

Considering the right-hand side of Eq. (64) term by term, for the first term, we have

$$
\begin{aligned}
& \left\langle\dot{b}_{l}^{*}(t) b_{m}(t) b_{s}(t) b_{k}^{*}(0)\right\rangle \Delta_{m s}^{k l} \\
& =\left\langle\left[ 2 \imath \sum_{\alpha, \beta, \gamma}^{\prime} \tilde{T}_{\beta \gamma}^{l \alpha} b_{\alpha}(t) b_{\beta}^{*}(t) b_{\gamma}^{*}(t)\right.\right. \\
& \left.\left.\times e^{-\imath \omega_{\beta \gamma}^{l \alpha}} \Delta_{\beta \gamma}^{l \alpha}\right] b_{m}(t) b_{s}(t) b_{k}^{*}(0)\right\rangle \Delta_{m s}^{k l} .
\end{aligned}
$$

We can use the near-Gaussian assumption to split the correlator of the sixth order in Eq. (65) into the product of three correlators of the second order, namely,

$$
\begin{aligned}
& \left\langle b_{k}^{*}(0) b_{m}(t) b_{s}(t) b_{\alpha}(t) b_{\beta}^{*}(t) b_{\gamma}^{*}\right\rangle \Delta_{m s}^{k l} \\
& =C_{k}(t) n_{m} n_{s} \delta_{\alpha}^{k}\left(\delta_{\beta}^{m} \delta_{\gamma}^{s}+\delta_{\gamma}^{m} \delta_{\beta}^{s}\right),
\end{aligned}
$$

where we have used that $n_{m}=C_{m}(0)$. Then, Eq. (65) becomes

$$
\begin{aligned}
& \left\langle\dot{b}_{l}^{*}(t) b_{m}(t) b_{s}(t) b_{k}^{*}(0)\right\rangle \Delta_{m s}^{k l} \\
& =4 \imath \tilde{T}_{m s}^{l k} C_{k}(t) n_{m} n_{s} e^{-\imath \tilde{\omega}_{m s}^{l k}} \Delta_{m s}^{k l} .
\end{aligned}
$$

Similarly, for the remaining two terms in Eq. (64), we have

$$
\begin{aligned}
& \left\langle b_{l}^{*}(t) \dot{b}_{m}(t) b_{s}(t) b_{k}^{*}(0)\right\rangle \Delta_{m s}^{k l} \\
& =-4 \imath \tilde{T}_{k l}^{m s} C_{k}(t) n_{l} n_{s} e^{\imath \tilde{\omega}_{k l}^{m s}} \Delta_{m s}^{k l},
\end{aligned}
$$

and

$$
\begin{aligned}
& \left\langle b_{l}^{*}(t) b_{m}(t) \dot{b}_{s}(t) b_{k}^{*}(0)\right\rangle \Delta_{m s}^{k l} \\
& =-4 \imath \tilde{T}_{k l}^{m s} C_{k}(t) n_{l} n_{m} e^{\imath \tilde{\omega}_{k l}^{m s}} \Delta_{m s}^{k l},
\end{aligned}
$$

respectively. Combining Eqs. (66), (67), and (68) with Eq. (64), we obtain

$$
\begin{aligned}
\dot{J}_{m s}^{k l}(t) \Delta_{m s}^{k l}= & 4 \imath \tilde{T}_{m s}^{k l} C_{k}(t) e^{-\imath \tilde{\omega}_{m s}^{k l} t} \Delta_{m s}^{k l} \\
& \times\left(n_{m} n_{s}-n_{l} n_{m}-n_{l} n_{s}\right) .
\end{aligned}
$$

Equation (69) can be solved for $J_{m s}^{k l}(t)$ under the assumption that the term $e^{-\imath \tilde{\omega}_{m s}^{k l} t}$ oscillates much faster than $C_{k}(t)$. We numerically verify [Fig. 9 below] the validity of this assumption of time-scale separation. Under this approximation, the solution of Eq. (69) becomes

$$
\begin{aligned}
J_{m s}^{k l}(t) \Delta_{m s}^{k l}= & 4 \tilde{T}_{m s}^{k l} C_{k}(t) \Delta_{m s}^{k l} \frac{e^{-\imath \tilde{\omega}_{m s}^{k l} t}-1}{-\tilde{\omega}_{m s}^{k l}} \\
& \times\left(n_{m} n_{s}-n_{l} n_{m}-n_{l} n_{s}\right)
\end{aligned}
$$


Plugging Eq. (70) into Eq. (61), we obtain the following equation for $C_{k}(t)$

$$
\begin{aligned}
\dot{C}_{k}(t)= & 8 C_{k}(t) \sum_{l, m, s}^{\prime}\left(\tilde{T}_{m s}^{k l}\right)^{2} \Delta_{m s}^{k l} \frac{1-e^{\imath \omega_{m s}^{k l} t}}{\omega_{m s}^{k l}} \\
& \times\left(n_{m} n_{s}-n_{l} n_{s}-n_{l} n_{m}\right) .
\end{aligned}
$$

Since in the thermal equilibrium $n_{k}$ is known, i.e., $n_{k}=$ $\left\langle\left|b_{k}(t)\right|^{2}\right\rangle=\theta / \tilde{\omega}_{k}$ [Eq. (26)], Eq. (71) becomes a closed equation for $C_{k}(t)$. The solution of Eq. (71) yields the autocorrelation function $C_{k}(t)$

$$
\begin{aligned}
\ln \frac{C_{k}(t)}{C_{k}(0)}= & 8 \sum_{l, m, s}^{\prime}\left(\tilde{T}_{m s}^{k l}\right)^{2} \frac{e^{\imath \omega_{m s}^{k l} t}-1-\imath \omega_{m s}^{k l} t}{\left(\omega_{m s}^{k l}\right)^{2}} \\
& \times\left(n_{l} n_{s}+n_{l} n_{m}-n_{m} n_{s}\right) \Delta_{m s}^{k l} .
\end{aligned}
$$

Using this observation, together with Eq. (56), finally, we obtain for the thermalized $\beta$-FPU chain

$$
\begin{aligned}
\ln \frac{C_{k}(t)}{C_{k}(0)}= & \frac{9 \beta^{2} \theta^{2}}{8 N^{2} \eta^{6}} \omega_{k} \sum_{l, m, s}^{\prime}\left(\omega_{m}+\omega_{s}-\omega_{l}\right) \Delta_{m s}^{k l} \\
& \times \frac{e^{\imath \omega_{m s}^{k l} t}-1-\imath \omega_{m s}^{k l} t}{\left(\omega_{m s}^{k l}\right)^{2}} .
\end{aligned}
$$

Equation (73) gives a direct way of computing the correlation function of the renormalized waves $\tilde{a}_{k}$, which, in turn, allows us to predict the spatiotemporal spectrum $\left|\hat{a}_{k}(\omega)\right|^{2}$. In Fig. 6(a), we plot the analytical prediction (via Eq. (73)) of the spatiotemporal spectrum $\left|\hat{a}_{k}(\omega)\right|^{2} \equiv\left|b_{k}\left(\omega-\tilde{\omega}_{k}\right)\right|^{2}=\mathfrak{F}^{-1}[C(t)]\left(\omega-\tilde{\omega}_{k}\right)$. By comparing this plot with the one presented in Fig. 6(b), in which the corresponding numerically measured spatiotemporal spectrum is shown, it can be seen that the analytical prediction of the frequency spectrum via Eq. (73) is in good qualitative agreement with the numerically measured one. However, to obtain a more detailed comparison of the analytical prediction with the numerical observation, we show, in Fig. 7, the numerical frequency spectra of selected wave modes with the corresponding analytical predictions. It can be clearly observed that the agreement is rather good. One of the important characteristics of the frequency spectrum is the width of the spectrum. We compute the width $W(f)$ of the spectrum $f(\omega)$ by

$$
W(f)=\frac{\int f(\omega) d \omega}{\max _{\omega} f(\omega)}
$$

In Fig. 8, we compare the width, as a function of the wave number $k$, of the frequency peaks from the numerical observation with that obtained from the analytical predictions. We observe that, for weak nonlinearity $(\beta=0.125)$, the analytical prediction and the numerical observation are in excellent agreement. In the weakly nonlinear regime, this agreement can be attributed to the validity of (i) the near-Gaussian assumption, and (a)

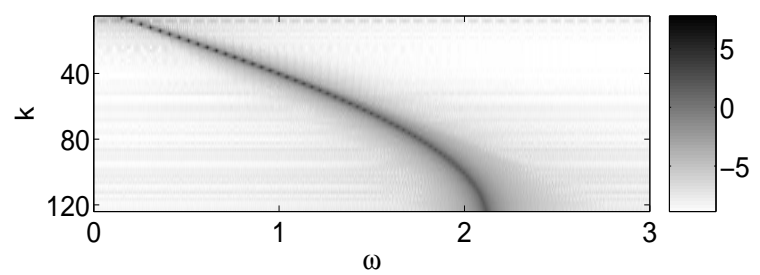

(b)

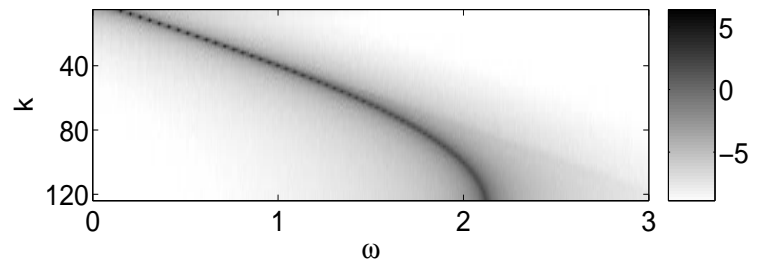

FIG. 6: (a) Plot of the analytical prediction for the spatiotemporal spectrum $\left|\hat{a}_{k}(\omega)\right|^{2}$ via Eq. (73). (b) Plot of the numerically measured spatiotemporal spectrum $\left|\hat{a}_{k}(\omega)\right|^{2}$. The parameters in both plots were $N=256, \beta=0.125, E=100$ and $\eta=1.06, \theta=0.401$. $\eta$ and $\theta$ were computed analytically via Gibbs measure. The darker gray scale correspond to larger values of $\left|\hat{a}_{k}(\omega)\right|^{2}$ in $\omega-k$ space. $\left[\max \left\{-8, \ln \left|\hat{a}_{k}(\omega)\right|^{2}\right\}\right.$ is plotted for clear presentation].

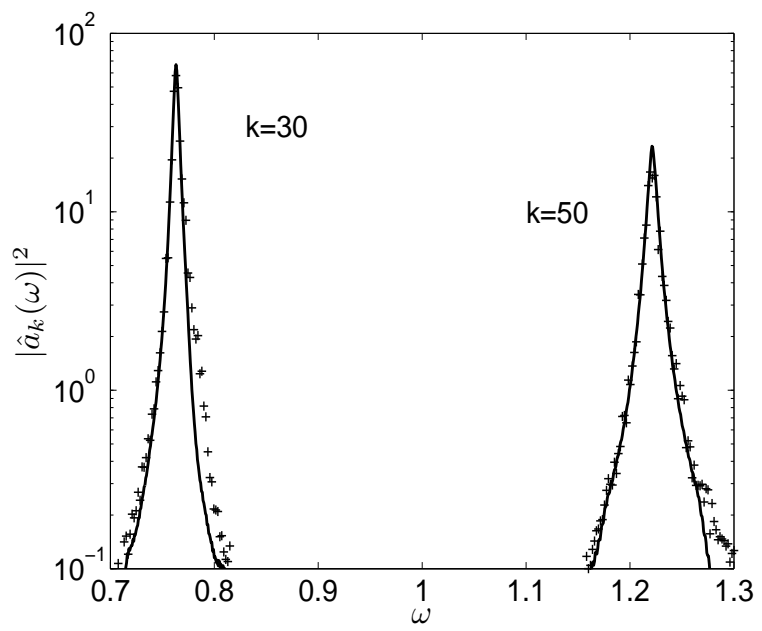

FIG. 7: Temporal frequency spectrum $\left|\hat{a}_{k}(\omega)\right|^{2}$ for $k=30$ (left peak) and $k=50$ (right peak). The numerical spectrum is shown with pluses and the analytical prediction [via Eq. (73)] is shown with solid line. The parameters were $N=256$, $\beta=0.125, E=100$.

(ii) the separation between the linear dispersion time scale and the time scale of the correlation $C_{k}(t)$. This separation was used in deriving the analytical prediction [Eq. (73)]. However, when the nonlinearity becomes larger $(\beta=0.25$ and $\beta=0.5)$, the discrepancy between the numerical measurements and the analytical prediction increases, as can be seen in Fig. 8. Nevertheless, it is important to emphasize that, even for very strong nonlinearity, our prediction is still qualitatively valid, as seen in Fig. 8. In order to find out the effect of the umk- 


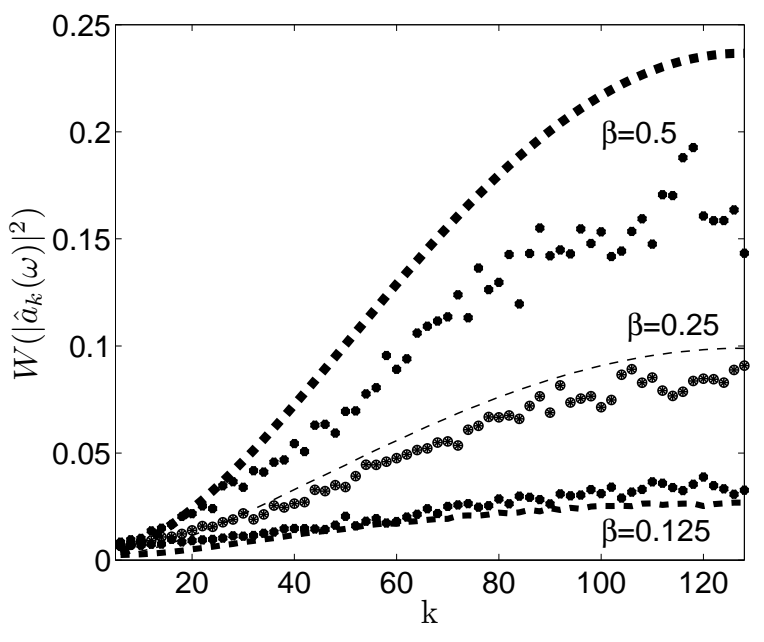

FIG. 8: Frequency peak width $W\left(\left|\hat{a}_{k}(\omega)\right|^{2}\right)$ as a function of the wave number $k$. The analytical prediction via Eq. (73) is shown with a dashed line and the numerical observation is plotted with solid circles. The parameters were $N=256, E=$ 100. The upper thick lines correspond to $\beta=0.5$, the middle fine lines correspond to $\beta=0.25$, and the lower solid circle and dashed line (almost overlap) correspond to $\beta=0.125$.

lapp scattering due to the finite size of the chain, we also computed the correlation [Eq. (73)] with the "conventional" $\delta$-function $\delta_{m s}^{k l}$ (i.e., without taking into account the umklapp processes) instead of our "periodic" delta function $\Delta_{m s}^{k l}$. It turns out that the correlation time is approximately $30 \%$ larger if it is computed without umklapp processes taken into account for the case $N=256$, $\beta=0.5, E=100$. It demonstrates that the influence of the non-trivial umklapp resonances is important and should be considered when one describes the dynamics of the finite length chain of particles. Finally, in Fig. 9, we verify the time scale separation assumption used in our derivation, i.e., the correlation time of the wave mode $k$ is sufficiently larger than the corresponding linear dispersion period $\tilde{t}_{k}=2 \pi / \tilde{\omega}_{k}$. In the case of small nonlinearity $(\beta=0.125)$, the two-point correlation changes over much slower time scale than the corresponding linear oscillations - the correlation time is nearly two orders of magnitude larger than the corresponding linear oscillations for weak nonlinearity $\beta=0.125$, and nearly one order of magnitude larger than the corresponding linear oscillations for stronger nonlinearity $\beta=0.25$ and $\beta=0.5$. This demonstrates that the renormalized waves have long lifetimes, i.e., they are coherent over time-scales that are much longer than their oscillation time-scales.

\section{CONCLUSIONS}

We have studied the statistical behavior of the nonlinear periodic lattice with the nearest neighbor interactions in thermal equilibrium. We have extended the notion of normal modes to the nonlinear system by showing that

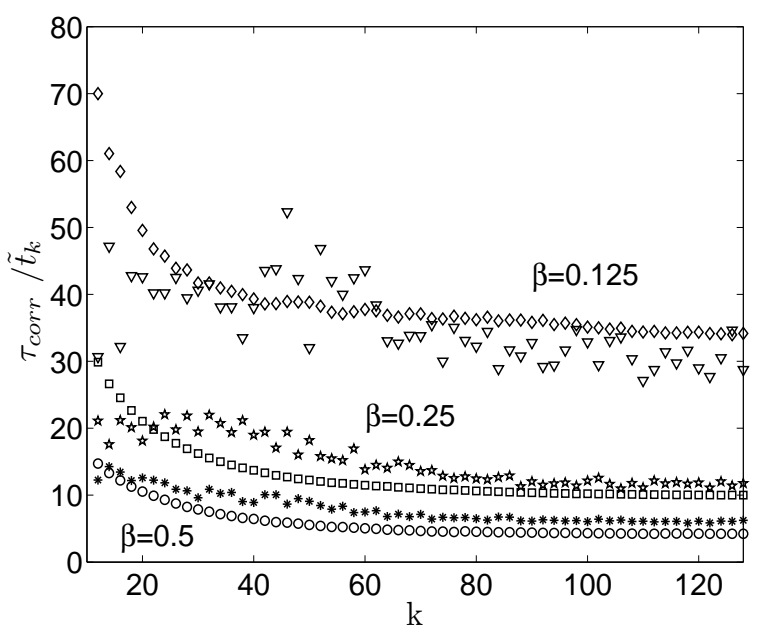

FIG. 9: Ratio, as a function of $k$, of the correlation time $\tau_{k}$ of the mode $k$ to the corresponding linear period $\tilde{t}_{k}=2 \pi / \tilde{\omega}_{k}$. Circles, squares, and diamonds represent the analytical prediction for $\beta=0.5, \beta=0.25$, and $\beta=0.125$ respectively. Stars, pentagrams, and triangles correspond to the numerical observation for $\beta=0.5, \beta=0.25$, and $\beta=0.125$ respectively. The parameters were $N=256, E=100$. The ratio is sufficiently large for all wave numbers $k$ even for relatively large $\beta=0.5$, which validates the time-scale separation assumption used in deriving Eq. (70). The comparison also suggests that for smaller $\beta$ the analytical prediction should be closer to the numerical observation, as is confirmed in Fig. 8.

regardless of the strength of nonlinearity, the system in thermal equilibrium can still be effectively characterized by a complete set of renormalized waves, in the sense that those renormalized waves possess the Rayleigh-Jeans distribution and vanishing correlations between different wave modes. In addition, we have studied the property of dispersion relation of the renormalized waves. The results we obtained in Section II are general and can be applied to the large class of nonlinear systems with the nearest neighbor interactions in thermal equilibrium.

We have further focused our attention on the particular system with the nearest neighbor interactions - the famous FPU chain. We have confirmed that the general renormalization framework that we discussed above is consistent with the numerical observations. In particular, we have shown that the renormalized dispersion of the thermalized $\beta$-FPU chain is in excellent agreement with the numerical one for a wide range of the nonlinearity strength. We have further demonstrated that the renormalized dispersion is a direct consequence of the trivial resonant interactions of the renormalized waves. Using a self-consistency argument, we have found an approximation of the renormalization factor via a meanfield approximation. In addition, we have used the multiple time-scale, statistical averaging method to obtain the theoretical prediction of the spatiotemporal spectrum and demonstrated that the renormalized waves have long lifetimes. We note that the results obtained here can be 
extended to general nonlinear potentials with the nearest neighbor interactions.

The scenario of the wave behavior in the thermal equilibrium we obtained here may suggest a theoretical framework for the application of the wave turbulence to $\beta$-FPU in the situation of near-equilibrium.

\section{Acknowledgments}

We thank Sergey Nazarenko and Naoto Yokoyama for discussions. Y.L. was supported by NSF CAREER DMS 0134955 and D.C. was supported by NSF DMS 0507901.

\section{APPENDIX A: LIMITING BEHAVIORS OF $\eta$ FOR THE THERMALIZED $\beta$-FPU CHAIN}

We change variables $y_{j}=q_{j}-q_{j+1}$ in the Hamiltonian (29) for $\beta$-FPU to obtain

$$
H(p, y)=\sum_{j=1}^{N}\left[\frac{1}{2} p_{j}^{2}+\frac{1}{2} y_{j}^{2}+\frac{\beta}{4} y_{j}^{4}\right]
$$

Next, we compute the pdf's for the momentum and displacement. Any $p_{j}$ is distributed with the Gaussian pdf $z_{p}=C_{p} \exp \left(-\theta^{-1} p^{2} / 2\right)$ and any $y_{j}$ is distributed with the pdf $z_{y}=C_{y} \exp \left(-\theta^{-1}\left(y^{2}+\beta y^{4} / 2\right) / 2\right)$, where $C_{p}$ and $C_{y}$ are the normalizing constants. As we have discussed, the renormalization factor $\eta$ of the $\beta$-FPU system in thermal equilibrium is given by Eq. (25), and its approximation via the self-consistency argument $\eta_{s c}$ is given by Eq. (52). Here, we compare the behavior of both formulas in two limiting cases, i.e., the case of small nonlinearity $\beta \rightarrow 0$ and the case of strong nonlinearity $\beta \rightarrow \infty$. We will use the following expressions for the average density of kinetic, quadratic potential and quartic potential parts of the total energy of the system

$$
\begin{aligned}
& \frac{\langle K\rangle}{N}=\frac{1}{N} \sum_{j=1}^{N} \frac{\left\langle p_{j}^{2}\right\rangle}{2}=\frac{1}{2} \theta, \\
& \frac{\langle U\rangle}{N}=\frac{1}{N} \sum_{j=1}^{N} \frac{\left\langle y_{j}^{2}\right\rangle}{2}=\frac{1}{2}\left\langle y^{2}\right\rangle, \\
& \frac{\langle V\rangle}{N}=\frac{\beta}{4 N} \sum_{j=1}^{N}\left\langle y_{j}^{4}\right\rangle=\frac{\beta}{4}\left\langle y^{4}\right\rangle .
\end{aligned}
$$

In a canonical ensemble, the temperature of a system is given by the temperature of the heat bath. By identifying the average energy density of the system with $\bar{e}=E / N$ in our simulation (a microcanonical ensemble), we can determine $\theta$ as a function of $\bar{e}$ and $\beta$ by the following equation

$$
\frac{1}{N}(\langle K\rangle+\langle U\rangle+\langle V\rangle)=\bar{e}
$$

We start with the case of small nonlinearity $\beta \rightarrow 0$. Suppose in the first order of the small parameter $\beta$ the temperature has the following form

$$
\theta(\beta)=\theta_{0}+\beta \theta_{1}
$$

where $\theta_{0}=O(1)$ and $\theta_{1}=O(1)$. We find the values of $\theta_{0}$ and $\theta_{1}$ using the constraint (A5). We use the following expansions in the small parameter $\beta$

$$
\begin{aligned}
& \int_{-\infty}^{\infty} e^{-\frac{1}{2 \theta(\beta)}\left(y^{2}+\beta \frac{y^{4}}{2}\right)} d y \\
& =\sqrt{\frac{\pi}{8}} \sqrt{\theta_{0}}\left(4+\left(\frac{2 \theta_{1}}{\theta_{0}}-3 \theta_{0}\right) \beta\right)+O\left(\beta^{2}\right), \\
& \int_{-\infty}^{\infty} y^{2} e^{-\frac{1}{2 \theta(\beta)}\left(y^{2}+\beta \frac{y^{4}}{2}\right)} d y \\
& =\sqrt{\frac{\pi}{8}} \sqrt{\theta_{0}}\left(4 \theta_{0}+\left(6 \theta_{1}-15 \theta_{0}^{2}\right) \beta\right)+O\left(\beta^{2}\right), \\
& \int_{-\infty}^{\infty}\left(y^{2}+\frac{\beta}{2} y^{4}\right) e^{-\frac{1}{2 \theta(\beta)}\left(y^{2}+\beta \frac{y^{4}}{2}\right)} d y \\
& =\sqrt{\frac{\pi}{8}} \sqrt{\theta_{0}}\left(4 \theta_{0}+\left(6 \theta_{1}-9 \theta_{0}^{2}\right) \beta\right)+O\left(\beta^{2}\right) .
\end{aligned}
$$

Then, in the first order in $\beta$, Eq. (A5) becomes

$$
\theta_{0}+\beta \theta_{1}+\frac{\sqrt{\frac{\pi}{8}} \sqrt{\theta_{0}}\left(4 \theta_{0}+\left(6 \theta_{1}-9 \theta_{0}^{2}\right) \beta\right)}{\sqrt{\frac{\pi}{8}} \sqrt{\theta_{0}}\left(4+\left(\frac{2 \theta_{1}}{\theta_{0}}-3 \theta_{0}\right) \beta\right)}=2 \bar{e}
$$

and we obtain $\theta_{0}=\bar{e}$ and $\theta_{1}=(3 / 4) \bar{e}^{2}$. Therefore, for the average kinetic energy density, we have

$$
\frac{\langle K\rangle}{N}=\frac{1}{2} \bar{e}+\frac{3}{8} \bar{e}^{2} \beta+O\left(\beta^{2}\right)
$$

and, for the average quadratic potential energy density, we have

$$
\begin{aligned}
\frac{\langle U\rangle}{N} & =\frac{1}{2} \frac{\sqrt{\frac{\pi}{8}} \sqrt{\theta_{0}}\left(4 \theta_{0}+\left(6 \theta_{1}-15 \theta_{0}^{2}\right) \beta\right)}{\sqrt{\frac{\pi}{8}} \sqrt{\theta_{0}}\left(4+\left(\frac{2 \theta_{1}}{\theta_{0}}-3 \theta_{0}\right) \beta\right)} \\
& =\frac{1}{2} \bar{e}-\frac{9}{8} \bar{e}^{2} \beta+O\left(\beta^{2}\right) .
\end{aligned}
$$

Finally, we obtain Eq. (53), i.e., for small $\beta$

$$
\eta=1+\frac{3}{2} \bar{e} \beta+O\left(\beta^{2}\right) .
$$

Similarly, from Eq. (52), we find the small $\beta$ limit of the approximation $\eta_{s c}$

$$
\eta_{s c}=1+\frac{3}{2} \bar{e} \beta+O\left(\beta^{2}\right) .
$$

Now, we consider the case of strong nonlinearity $\beta \rightarrow$ $\infty$. From Eq. (A5), we conclude that temperature in the 
large $\beta$ limit, which we denote as $\theta_{\infty}$, stays bounded, i.e., $0<\theta_{\infty}<2 \bar{e}$, and, in the limit of large $\beta$, we obtain for Eq. (A5)

$$
\theta_{\infty}+\frac{\int_{-\infty}^{\infty} \frac{\beta}{2} y^{4} e^{-\frac{\beta}{4 \theta \infty} y^{4}} d y}{\int_{-\infty}^{\infty} e^{-\frac{\beta}{4 \theta \infty} y^{4}} d y}=2 \bar{e}
$$

After performing the integration, we obtain $\theta_{\infty}=(4 / 3) \bar{e}$, and the average kinetic energy density becomes $\langle K\rangle / N=$ $(2 / 3) \bar{e}$. For the average quadratic potential energy density, we have

$$
\frac{\langle U\rangle}{N}=\frac{1}{2} \frac{\int_{-\infty}^{\infty} y^{2} e^{-\frac{\beta}{4 \theta \infty} y^{4}} d y}{\int_{-\infty}^{\infty} e^{-\frac{\beta}{4 \theta \infty} y^{4}} d y}=\frac{\Gamma\left(\frac{3}{4}\right)}{\Gamma\left(\frac{1}{4}\right)}\left(\frac{4 \bar{e}}{3 \beta}\right)^{\frac{1}{2}}
$$

For the renormalization factor, we obtain the following large $\beta$ scaling

$$
\eta=\sqrt{\frac{\Gamma\left(\frac{3}{4}\right)}{\sqrt{3} \Gamma\left(\frac{1}{4}\right)}} \bar{e}^{\frac{1}{4}} \beta^{\frac{1}{4}} .
$$

Similarly, for the approximation of $\eta_{s c}$, we obtain $A=$ $C \sqrt{\bar{e} \beta}, B=4 \bar{e} \beta$, and $C=2 \sqrt{3} \Gamma(3 / 4) / \Gamma(1 / 4)$. Therefore, the large $\beta$ scaling of $\eta_{s c}$ becomes

$$
\eta_{s c}=\sqrt{\frac{C+\sqrt{C^{2}+16}}{2}} \bar{e}^{\frac{1}{4}} \beta^{\frac{1}{4}}
$$

which yields Eq. (54).
[1] Focus issue: The Fermi-Pasta-Ulam problem, the first 50 years, Chaos 15 (2005).

[2] M. Peyrard, Nonlinearity 17, R1 (2004), and references therein.

[3] M. Toda, Theory of Nonlinear Lattice (Springer-Verlag, New York, 1989).

[4] L. Landau and E. Lifshitz, Statistical Physics, Course of Theoretical Physics Vol. 5 (Pergamon, Oxford, 1981).

[5] V.E. Zakharov, V.S. Lvov, and G. Falkovich, Kolmogorov Spectra of Turbulence (Springer-Verlag, Berlin, 1992).

[6] C. Alabiso, M. Casartelli, P. Marenzoni, J. Stat. Phys. 79, 451 (1995).

[7] S. Lepri, Phys. Rev. E 58, 7165 (1998)

[8] U. Zülicke and A. H. MacDonald, Physica (Amsterdam) 6E, 104 (2000) and references therein.

[9] J. Biello, P. Kramer, Y. Lvov, Proc. of the fourth international conf. on dyn. sys. and diff. eqns., 113 (2001); P. Kramer, J. Biello, Y. Lvov, ibid., 482 (2003).

[10] L. Landau and E. Lifshitz, Mechanics, Course of Theoretical Physics Vol. 1 (Pergamon, Oxford, 1976).

[11] A. J. Lichtenberg and M. A. Lieberman, Regular and Chaotic Dynamics, 2nd ed. (Springer-Verlag, Berlin, 1992).

[12] B. Gershgorin, Y.V. Lvov, D. Cai, Phys. Rev. Lett. 95, $264302(2005)$.

[13] E. Fermi, J. Pasta, and S. Ulam, Los Alamos Scientific Laboratory Report No. LA-1940 (reprinted in Fermi E. Collected papers by University of Chicago Press, Chicago, 1965, Vol II, p 978).

[14] J. Ford, Phys. Rep., 213, 271, (1992).
[15] C. Alabiso and M. Casartelli, J. Phys. A: Math. Gen. 34, 1223 (2001).

[16] S. Lepri, R. Livi, A. Politi, Chaos 15, 015118 (2005).

[17] A. Carati, L. Galgani, A. Giorgilli, Chaos 15, 015105 (2005).

[18] P. Poggy and S. Ruffo, Physica (Amsterdam) 103D, 251 (1997).

[19] H. Yoshida, Phys. Lett. A 150, 262 (1990).

[20] G. Parisi, Europhysics Lett. 40, 357 (1997).

[21] T. Cretegny et al., Physica (Amsterdam) 121D, 109 (1998).

[22] A. Newell, S. Nazarenko, and L. Biven, Physica (Amsterdam) 152D, 520 (2001)

[23] Y.V. Lvov and S. Nazarenko, Phys. Rev. E 69, 066608 (2004); Y. Choi, Y.V. Lvov, S. Nazarenko, and B. Pokorni, Phys. Lett. A 339, 361 (2005).

[24] D. Cai et al., Physica (Amsterdam) 152D, 551 (2001).

[25] A.J. Majda, D.W. McLaughlin, and E.G. Tabak, J. Nonlinear Sci. 6, 9 (1997).

[26] B. Rink, Comm. Math. Phys. 218(3), 665 (2001).

[27] B. Rink, Physica (Amsterdam) 175D, 31 (2003).

[28] Y.V. Lvov, S. Nazarenko, B. Pokorni, Physica (Amsterdam) 218D, 24 (2006).

[29] C. Connaughton, S.V. Nazarenko, and A.N. Pushkarev, Phys. Rev. E. 63, 046306 (2001).

[30] A. Pushkarev, Eur. J. Mech. B/fluids 18(3), 345 (1999).

[31] J. Benney and A. Newell, Stud. in Appl. Math. 48, 29 (1969). 